\title{
IV. DIE VERWANDLUNG
}

Als Anna d'Este Anfang Oktober 1548 Eltern, Geschwister und Heimatstadt verließ, war sie eine junge Frau von kaum siebzehn Jahren, vor allem aber war sie die Tochter des Herzogs von Ferrara und somit, trotz Einfluß der Mutter, eine Italienerin, die italienisch sprach, italienisch schrieb und sich italienisch kleidete. Als sie zwei Monate später in Paris anlangte, war sie zur Französin geworden. Die Italiener und Italienerinnen ihres Gefolges waren zum großen Teil ausgetauscht, die Prinzessin selbst unterhielt sich auf Französisch und kleidete sich nach französischer Mode. Nur ihre an den Vater gerichteten Briefe waren auch weiterhin in dessen Sprache verfaßt und sollten es, von wenigen Ausnahmen abgesehen, all die Jahre uber bleiben. Neben der Erziehung am Hof der Mutter und der Tatsache, daß Anna von klein auf von französischer Sprache und Kultur geprägt war, ist ihre rasche Integration in die Hofgesellschaft um Heinrich II. und Katharina von Medici wohl der Verwandlung zuzuschreiben, die sich im Laufe ihrer langen, teils mühsamen, teils unterhaltsamen Reise in ihr vollzogen haben muß. Ercoles Botschafter, der die Prinzessin noch aus Ferrara kannte, mußte nach ihrer Ankunft in Paris feststellen, ch'ella non è più la princessa di Ferrara ma Madama di Umala'. Auch Annas Eingliederung in die Hofgesellschaft vollzog sich rasch und problemlos, was sicher zu einem großen Teil damit zusammenhing, daß sie die Tochter von Heinrichs Tante und somit seine Cousine ersten Grades war. Die Tatsache, französisches Blut in ihren Adern zu haben, fuhrte dazu, daß der neuen Herzogin von Aumale Ehren erwiesen wurden, wie sie sonst nur einer französischen Königin zugestanden worden wären, wie etwa anläßlich ihres Empfangs durch die Pariser Ratsherren, die ihr bis vor die Tore der Stadt entgegengingen, ein Privileg, das sonst nur souveränen Fürsten oder eben der Königin zugestanden wurde. Königlich auch ihre Hochzeitsfeier in SaintGermain-en-Laye, königlich die Geschenke; und der Rang, den Anna bei Hofe bekleiden sollte, ließ ebensowenig zu wünschen ubrig. Innerhalb kürzester Zeit war aus der Prinzessin von Ferrara eine französische Fürstin geworden.

${ }^{1}$ Giulio Alvarotti an Ercole d'Este, 5. Dez. 1548, Paris, in: ASM, Canc. duc., Amb. Francia 45 . 
1. „En voz adieux, dames, cessés voz pleurs« Die lange Reise nach Frankreich

\section{Der schwierige Abschied von der Mutter}

Renée de France hătte ihre ălteste Tochter gerne an den Hof von Frankreich begleitet, doch der Herzog gestattete ihr die Reise nur bis Mantua. Wegen der schlechten Straßen sollte die Prinzessin diese erste Etappe auf dem Wasser reisen, und Ercole ließ seinen Buzentaur, der von vier Pferden gezogen wurde, sowie einige andere Boote ausstatten und prächtig schmücken. Bereits am Tag vor der Abreise wurden neunzehn Kisten Reisegepäck auf Maultiere verladen und direkt in Richtung Mailand vorausgeschickt, der Rest wurde von einem Kutscher am Palazzo San Francesco abgeholt und nach Mantua gebracht ${ }^{2}$. Viel zu spät hatte der Herzog bei Ercole Gonzaga angefragt, ob die Reisegesellschaft unterwegs in dessen delizia in Sacchetta beherbergt werden könne. Seinen Unmut dissimulierend, antwortete der Kardinal, es sei ihm trotz der knappen Zeit fur die Vorbereitungen eine Ehre, die Braut zu empfangen. Dem Bruder gegenuber verschwieg er seinen Zorn uber das Verhalten des Herzogs von Ferrara jedoch nicht: che tratto è quello che ne fa il duca di Ferrara di venire qua in casa [mia] con tanti Sig. ${ }^{r}$ Francesi et la metà di Ferrara all' improviso. Am liebsten, so der Kardinal, wirde er ablehnen, et cosi lasciare andare quella sua sposa con la sua franciosaria dove la vuole alle sue.spese, che è quello che preme più all' amico ${ }^{3}$.

Anfang Oktober 1548 verließ Anna die Heimatstadt; ihre Eltern, Geschwister und fast die gesamte Bevölkerung Ferraras gaben ihr das Geleit:

Quasi tutte le persone, maschij e femine, piangevano per dolore della partita di questa signora, che veramente era la cortesia di Ferrara, e veramente questa signora era più amata da cadauna persona che qual si voglia donna che fusse in Ferrara, e questa sua partita parse che portasse via il core a cadauna persona, tanto era amata dal populo ${ }^{4}$.

Auch in zwei Liedern, die Cyprian de Rore der Prinzessin zum Abschied widmete, kommt der Kummer der Zuruckgebliebenen zum Ausdruck: En voz adieux, dames, cessés voz pleurs [...]. / Ne plourés plus de sa beaulté

${ }^{2}$ Ercole d'Este an Giulio Alvarotti, 5. Sept. u. 2. Okt. 1548, in: ASM, Canc. duc., Amb. Francia 39; Rechnungsbuch 1548-I (Okt). Vgl. FONTANA, Renata di Francia, Bd. 2, S. 291.

${ }^{3}$ Ercole Gonzaga an Ercole d'Este und Ferrante Gonzaga, 2. Okt. 1548, in: FonTANA, Renata di Francia, Bd. 2, S. 290. Das Wort in Klammern wurde von mir anhand ASMan, AG 1917 korrigiert.

${ }^{4}$ DA MARANO, Cronaca di Ferrara, fol. 11v. Vgl. den ersten Dialog von Lilio Gregorio Giraldi, in: GREGORIO GIRALDI, Dialoghi, S. 38: Omnis haec civitas tam illustrem ornatamque puellam minime libenter in Gallias proficisci videt. 
l'absence, / Dont vous pensés estre privez vos yeulx; die Antwort lautet: Hellas, comment, hellas, comment voulés vous que noz yeulx / Cessent leurs pleurs laissant telle princesse? ${ }^{5}$ Vom Vater verabschiedete sich Anna am Hafen. Dann bestieg sie, gemeinsam mit der Mutter, den Geschwistern und den Edeldamen, die sie auf ihrer Reise begleiten sollten, die Boote. Den ganzen Tag verbrachte die Gesellschaft auf dem Wasser, bis sie am späten Nachmittag Ficarolo erreichte, wo die Prinzessin von ihrem Bruder Alfonso Abschied nahm. Die folgende Nacht schliefen die Damen in Sacchetta und kamen am Tag darauf in Mantua an, wo sie drei Tage lang von ihrem Gastgeber und dem gesamten Adel der Stadt verwöhnt wurden ${ }^{6}$. Der anfängliche Ärger des Kardinals war bald verflogen, und er zeigte sich zufrieden über den Besuch aus Ferrara, vor allem aber über die junge Braut, la quale certo è una ben creata $S .^{r a}$ di conveniente bellezza, ma sopra tutto di boniss. ${ }^{a}$ gratia. Renée, um den bevorstehenden Abschied hinauszuzögern, tătigte in Mantua die letzten Besorgungen furr Anna und hielt die Reisegesellschaft so lange wie moglich auf. Doch schließlich, am 8. Oktober, trennten sich Mutter und Tochter unter Tränen, und die Herzogin kehrte zu den, wie sie traurig feststellte, deulx filles qui me sont encores demeurées nach Ferrara zurück?

Wie schwer Renée der Abschied fiel, ist aus der großen Anzahl Briefe zu ersehen, die sie an die verschiedenen Reisestationen der Prinzessin sandte. Noch von Mantua aus machte sich einer ihrer Diener mit einem ersten Schreiben auf den Weg, und der Postmeister von Bologna wurde wiederholt entlohnt, pour faire porter en dilligence et sur chevaulx de poste ung pacquet de lettres [...] à Madame la duchesse d'Aumalle ${ }^{8}$. Auch tauschte Anna regelmäBig Briefe mit dem Vater aus, dem sie zu Beginn ihrer Reise versprochen hatte, ihn uber alle Vorkommnisse zu unterrichten, und jeden zweiten Tag erhielt sie Post von den Kardinälen von Ferrara und von Guise. Letzterer hatte die Bedeutung, die der schriftliche Austausch für die junge Braut hatte, erkannt, und er forderte den Bruder auf, seiner Braut oft zu schreiben. Tatsächlich scheint Anna großen Wert vor allem auf die an ihren zukünttigen Gemahl gerichteten Schreiben gelegt zu haben, einem ihrer Reisebegleiter verriet sie, sie schriebe lieber hundert andere Briefe als einen an ihn, aus Angst, etwas Falsches zu schreiben. Erhielt sie dann eine Antwort, war die Prinzessin fort

${ }^{5}$ Cyprian de Rore, En voz adieux - Hellas, comment, in: RORE, Opera, Bd. 8, S. 39-43.

- DA MARANO, Cronaca di Ferrara, fol. 12r; ISNARDI, Ricordi diversi, S. 114; Rechnungsbuch 1548-I (Okt.).

${ }^{7}$ Ercole Gonzaga an Ferrante Gonzaga, 7. Okt. 1548, in: FONTANA, Renata di Francia, Bd. 2, S. 290; Renée de France an Ercole d'Este, 15. Nov. (1548), Consandolo, in: ASM, Casa 155, fol. 164r. Zu den Verzögerungen der Weiterreise: Rechnungsbuch 1548-II (Okt.); Louis de Lorraine an François de Lorraine, 3. Nov. (1548), in: BnF, Fr. 20467, S. 73f.

${ }^{8}$ Rechnungsbuch 1548-II (Okt.). Die Briefe gingen u.a. nach Turin und Grenoble. Der Briefwechsel jener Jahre ist nicht erhalten. 
ayse, sie rief die Mitglieder ihres Gefolges zusammen, las ihnen die Briefe vor und sandte Kopien der Schreiben an ihre Mutter?.

\section{Mühsal und Annehmlichkeiten der Reise}

Innerhalb von zwei Monaten sollte der Brautzug eine Strecke von fast tausendfünfhundert Kilometern hinter sich bringen. Die Beschwerlichkeiten der Reise waren erheblich, nicht zuletzt der schlechten Straßen und des herbstlichen Wetters wegen. Die Begleiter klagten über den langen und mühsamen Weg, welcher sie über monti $e$ sassi und durch paese inabitabile führe, und hinsichtlich des Wetters berichteten sie sowohl von extremer Hitze als auch von stundenlangen Regenglissen ${ }^{10}$. Für Anna kam die durch den Abschied von den Eltern und der vertrauten Umgebung hervorgerufene Belastung hinzu. Florimond de Biencourt, der die Prinzessin von Beginn an auf ihrem Weg begleitete, war erleichtert, als die junge Frau sich an die neue Situation zu gewöhnen begann. Dem Bräutigam schrieb Biencourt, er danke Gott, daß sich seine zukünftige Gemahlin seit ihrer Abreise aus Mantua tapfer gehalten habe: Par avant elle ne faisoit que pleurer, mes [mais] je vous puis aseurer, Monseigneur, que depuis il n'a esté question sinon que de se rejouyr et parler souvent de vous ${ }^{11}$.

Den größten Teil der Strecke reiste Anna in einer Sänfte, während ihre Begleiter den Weg zu Pferde zurucklegten. Sie trug Kleider aus schwarzem, mit Gold- oder Silberfäden durchwirktem Samt, aus Atlasstoff oder Brokat, ihren Hals schmückten Perlen, ihre Taille Rubine ${ }^{12}$. Die Gesellschaft kam nur langsam voran, und oft wurde tagelang gerastet, da sich die junge Braut, derartige Strapazen nicht gewöhnt, erholen mußte. Nous l'avons faict sejourner quelques jours par les chemins, craignant le travail pour aultant qu'elle n'avoit acoustume d'aller par peis, schrieb der ständig besorgte Biencourt. Auch Ercole wies seine Leute an, der Prinzessin die Fahrt so angenehm wie möglich

9 Anna d'Este an Ercole d'Este, 9. Okt. 1548, Cane bei Cremona, in: ASM, Canc. duc., Cart. prin. est. 1568 A14; Alfonso Gilioli an dens., 14. u. 27. Okt. 1548, Vercelli, Gap, in: Ibid. Amb. Francia 52; Germanico Savorgnano an dens., 27. Nov. 1548, Troyes, in: Ibid.; Charles de Lorraine an François de Lorraine, 18. Okt. 1548, Moulins, in: LoRRAINE, Lettres, S. 116; (Florimond) de Biencourt an dens., 25. Okt. (1548), Embrun, in: BnF, Fr. 20555, fol. 153r. Das Zitat: (François) de Hangest an dens., 25. Okt. (1548), Embrun, in: Ibid. fol. 155r.

${ }^{10}$ Alfonso Gilioli und Ferrante Trotti an Ercole d'Este, 10. u. 27. Okt. 1548, Cremona, Gap, in: ASM, Canc. duc., Amb. Francia 52.

${ }^{11}$ (Florimond) de Biencourt an François de Lorraine, 25. Okt. (1548), Embrun, in: BnF, Fr. 20555, fol. 153r. Vgl. Ferrante Trotti an Ercole d'Este, 10. Okt. 1548, Cremona, in: ASM, Canc. duc., Amb. Francia 52: La Signora principessa sta bene e si va conffortando per strada.

12 Alfonso Gilioli und Germanico Savorgnano an Ercole d'Este, 14. u. 19. Okt., 6. Nov. 1548, Vercelli, Turin, Lyon, in: ASM, Canc. duc., Amb. Francia 52. 
$\mathrm{zu}$ bereiten, ihr alle notwenigen Bequemlichkeiten zu verschaffen und dafür $\mathrm{zu}$ sorgen, che si conddussa questa signora sana et allegra, il quale obietto è principalissimo, e quando questo rieschi, tutto il restante si ha a riputar' niente $^{13}$. Doch erst als die Alpen uberschritten waren, fand sich für Anna die Möglichkeit, die Sänfte zu verlassen, ihren Zelter zu besteigen und die Zeit mit der Jagd zu verbringen. Den guten Humor scheint sie trotz aller Beschwernisse nicht verloren $\mathrm{zu}$ haben, und es wurde viel gelacht: Pour ceste heure elle est bien punye qu'elle n'oze rire, pour se que le froit luy a faict mal à la bouche, schrieb Biencourt, und das Gerücht, die Prinzessin sei schwermutig, wußte er entschieden zu dementieren: Je vous aseure, Monseigneur, qu' elle l'est aussy peu que fame que j'ay point veu' 14 .

Die Reisegruppe, welche Anna bis Grenoble begleitete, zählte mehr als vierhundert Personen und dreihundertfünfzig Pferde, eine kleine Zahl, wenn man sie mit den sechshundertsiebzig Vierhüflern vergleicht, die 1493 die Aussteuer der Bianca Maria Sforza über die Alpen getragen hatten, aber eine große Zahl, verglichen mit dem nur siebzig Personen und etwa zweihundert Pferde zăhlenden Brautzug der Barbara Gonzaga im Jahre $1474^{15}$. Hinsichtlich Unterbringung und Verpflegung brachte eine Reise mit derart großem Gefolge nicht wenige Schwierigkeiten mit sich. Innerhalb der Ortschaften fand sich oft nicht genügend Platz, und es fehlte an Bequemlichkeiten, so daß Ferrante Trotti, der für die Reise bis Grenoble verantwortlich war, sich beim Herzog über die enormen Probleme der Unterbringung beklagte, talmente che mi bisogna haver' gran patienza e gran destrezza pur' con tutte queste difficultà. Besonders in den kleinen, abgelegenen Bergdörfern der Alpen war es schwierig, Lebensmittel aufzutreiben, wie etwa auf dem Weg von Embrun nach Gap, den die Reisenden an einem Freitag zurücklegten: Semo poi gionti ne la più sparea vila, più diserta che sia possibille de veder'. E per più nostro vantaggio semo gionti di giorno da magro, che non se ritrova cosa del mondo da manzare ${ }^{16}$.

${ }^{13}$ (Florimond) de Biencourt an François de Lorraine, 3. Nov. (1548), Morey, in: BnF, Fr. 20553, fol. 7r; Ercole d'Este an Ferrante Trotti, 16. Okt. 1548, in: ASM, Canc. duc., Amb. Francia 52; Ferrante Trotti an Ercole d'Este, 14. Okt. 1548, Vercelli, in: Ibid.

${ }^{14}$ (Florimond) de Biencourt an François de Lorraine, 25. Okt. (1548), Embrun, in: BnF, Fr. 20555, fol. 154r.

${ }^{15}$ Karl-Heinz SPIESS, Unterwegs zu einem fremden Ehemann. Brautfahrt und Ehe in europäischen Fürstenhäusern des Spätmittelalters, in: Irene ERFEN, DERS. (Hg.), Fremdheit und Reisen im Mittelalter, Stuttgart 1997, S. 17-36, hier S. $26 f$.

${ }^{16}$ Alfonso Gilioli an Ercole d'Este, 27. Okt. 1548, Gap, in: ASM, Canc. duc., Amb. Francia 52; Ferrante Trotti an dens., 14. Okt. 1548, Vercelli, in: Ibid. Hier auch der Hinweis auf die Größe der Gruppe. Vgl. den Brief vom 10. Okt. 1548, Cremona, in: Ibid.: Lo alloggiamento è stato in casa dei Trecchi, li quali solo della stanza si hanno accommodato, che di nelle prestanze habbiamo provisto noi. No so come siamo stati visti con buon occhio e con cosi grassa compagnia in questa terra. So bene che son' stati mezo giorno prima che habbi- 
In der Regel wurde die Prinzessin aber in den Orten, durch die sie kam, mit allen Ehren empfangen. Pannen, wie sie sich etwa während der Brautfahrt der Yolande de Lorraine im Jahre 1497 ereigneten, als die Mönche eines als Herberge vorgesehenen Klosters der Reisegesellschaft die Aufnahme verweigerten, blieben den Ferraresen erspart ${ }^{17}$. In Vercelli ließ der Herzog von Savoyen Anna mit Freudenschüssen begrüßen, und sie schrieb nach Ferrara, che certo era cosa impossibile a farsi più honore e favore che S.E. sia fatto. In Chivasso bereitete der Gouverneur Ludovico da Birago der Prinzessin einen Empfang, quanto se 'l re vi fosse entrato, und in Turin erwartete sie der Prinz von Melfi mit einer großen Kompanie bewaffneter und unbewaffneter Reiter, die der Braut die Reverenz erwiesen ${ }^{18}$. In Frankreich, wo Annas Ankunft come del Messia erwartet wurde, gestaltete sich ihr Eintreffen nicht weniger spektakulär: Depuis qu'elle a esté arrivée dedens le peis du roy, elle a estée aultant honorablement reçeue que sy c'eust esté la raine. Die Guise hatten in Briançon, Embrun und Gap für den standesgemäßen Empfang der Herzogin von Aumale gesorgt, und in Grenoble, wo Anna ihren Schwiegervater treffen sollte, waren Wohnräume für die Gäste tapeziert und möbliert worden, affin qu'elle soit honorée et traictée ainsy qu'il luy apartyent ${ }^{19}$.

Auch beschenkten Claude de Lorraine und sein Sohn die Prinzessin und ihre Begleiter großzügig. Bereits in Briançon hatte der Herzog von Aumale seiner Braut verschiedene Gaben uberreichen lassen, darunter ein mit kunstvollen Miniaturen versehenes kleines Gebetbuch, dessen goldene Deckel mit Diamanten und Rubinen geschmuckt und mit den Buchstaben $" A \lll$ und $» F$ « gekennzeichnet waren. Über die der Prinzessin zugedachten Diamantringe berichtete Biencourt: Elle y a pris fort grant plaisir, et aussy qu'elle avoit grant besoing [...], car elle a fort belle main. In Grenoble uberreichte der Herzog von Guise seiner Schwiegertochter weitere Edelsteine und Perlen und erwies ihr und ihrem Gefolge alle erdenklichen Aufmerksamkeiten ${ }^{20}$.

no assignato lo alloggiamento a forieri et havevano consultato di non lasciarci entrare, se ben poi si son' rissoluti in contrario.

${ }^{17}$ Heidelore BOECKER, Hofe und Residenzen im spätmittelalterlichen Reich. Bilder und Begriffe, in: Mitteilungen der Residenzen-Kommission der Akademie der Wissenschaften zu Gortingen 14/1 (2004) S. 17-24, hier S. 21.

${ }^{18}$ Anna d'Este an Ercole d'Este, 15. Okt. 1548, Vercelli, in: ASM, Canc. duc., Cart. prin. est. $1568 \mathrm{~A} / 14$; Alfonso Gilioli an dens., 14. u. 19. Okt. 1548, Vercelli, Turin, in: Ibid. Amb. Francia 52.

${ }_{19}$ Girolamo Falletti an Ercole d'Este, 17. Okt. 1548, Lyon, in: ASM, Canc. duc., Amb. Francia 52; (Florimond) de Biencourt an François de Lorraine, 25. Okt. (1548), Embrun, in: BnF, Fr. 20555, fol. 153v.

${ }^{20}$ Alfonso Gilioli an Ercole d'Este, 27. Okt. 1548, Gap, in: ASM, Canc. duc., Amb. Francia 52; Anna d'Este an dens. (Nov. 1548), Grenoble, in: Ibid. Cart. prin. est. 1568 A/14. Das Zitat: (Florimond) de Biencourt an François de Lorraine, 25. Okt. (1548), Embrun, in: BnF, Fr. 20555, fol. 153r. 


\section{Der Austausch des Gefolges}

Annas Abschied von ihrer Stadt und ihrer Familie war in kleinen Schritten verlaufen. In Ferrara hatte sie dem Vater und den Ferraresen Lebewohl gesagt, in Ficarolo dem Bruder, und von der Mutter und den Schwestern war sie in Mantua ein letztes Mal in die Arme geschlossen worden. Nun war für die Prinzessin der Zeitpunkt des endgültigen Abschieds von ihrer Heimat und den Höfen der Eltern gekommen. Von Grenoble aus sollten ihre italienischen Begleiter nach Ferrara zurückkehren und sie selbst den Rest des Weges unter der Obhut der Guise zurlicklegen. Nur eine kleine Gruppe engster Vertrauter und Bediensteter, wie ihre Amme und ihr Diener Bernardino, sollten sie bis an den französischen Hof begleiten und erst einige Zeit später nach Ferrara zurückkehren. Die einzigen verbleibenden Ferrareser Edelleute waren Germanico Savorgnano, der umkehren wollte, nachdem er l'arrivée et les triomphes qui se feront gesehen habe, und Alfonso da Carpi, den Louis de Lorraine wăhrend der Reise in seinen Dienst ubernommen hatte ${ }^{21}$. Dem Vater schrieb die Prinzessin: Quanto a me, non ho voluto menar nissuno, per non far invidia a gli altri, e poi perché questi signori m'hanno fatto intender che non l'havevano a piacer. So ist auch hier die bei europäischen Fürstenhochzeiten ubliche Praktik zu beobachten, den Hofstaat der Braut bei ihrer Ankunft in ihrer neuen Heimat fast komplett auszutauschen, wie es etwa bei den Kaiserinnen oder Erzherzoginnen der Habsburger in der ersten Hälfte des 17. Jahrhunderts der Fall war. Doch scheint es den Guise in diesem Fall weniger um Fragen der Reputation und der Finanzen als vielmehr darum gegangen zu sein, ihr neues Familienmitglied von italienischen Einflüssen weitgehend fernzuhalten ${ }^{22}$.

$\mathrm{Zu}$ Annas neuer Ehrendame wurde Madame de Parroy emannt, die fortan zu den Verantwortlichen der Reise zählte, auf die sich der Herzog von Aumale,

${ }^{21}$ Ce sont ceulx et celles, fol. 2r. Annas Diener Bernardino kehrte drei Monate später nach Ferrara zuruck: Empfehlungsschreiben für Bernardino mio servitore von Anna d'Este an Ercole d'Este, 29. Jan. 1549, in: ASM, Canc. duc., Cart. prin. est. 1568 A/14. Es handelt sich wohl um denselben Bernardino oder Bernardin, der im Rechnungsbuch 1544, fol. 30r, 107r, $164 v$, im Gefolge von Renées Töchtern und in Messisbugos Kompendien der Jahre 15471548 al servicio della Signora principessa verzeichnet ist: CHIAPPINI, Corte, S. 134f. Wann Annas Amme nach Italien zurllckkehrte, ist unklar, ihr Empfehlungsschreiben ist nicht datiert. Im Herbst 1552 hielt sie sich noch in Frankreich auf: Anna d'Este an Ercole d'Este, 20. Aug. o.J., in: ASM, Canc. duc., Cart. prin. est. 1568 A/14; C'est le roole des personnes venans et mengeans en l'estat et maison de Madame la duchesse [douairière] de Guise, 1. Sept. 1552, in: BoUILLÉ, Histoire, Bd. 1, S. 550. Annas Reitknechte kehrten Anfang Mărz 1549 nach Italien zurück: Empfehlungsschreiben von Anna d'Este an Ercole d'Este, 2. März 1549, in: ASM, Canc. duc., Cart. prin. est. 1568 A/14. Darlber hinaus ist anzunehmen, daß der Verfasser der "Cronaca Da Marano« die Prinzessin an den Hof begleitete und erst nach dem Turnier im folgenden Jahr nach Italien zurückkehrte.

${ }^{22}$ Anna d'Este an Ercole d'Este, 1. (Nov. 1548), Grenoble, in: ASM, Canc. duc., Cart. prin. est. 1568 A/14. Vgl. Katrin KelLER, Hofdamen. Amtstraggerinnen im Wiener Hofstaat des 17. Jahrhunderts, Wien u.a. 2005, S. 36f., $68 f$. 
wie Florimond de Biencourt versicherte, voll und ganz verlassen könne ${ }^{23}$. Die Bedeutung der dame d'honneur für das Verhältnis zweier Jungvermählter kann an den vier langen Kapiteln abgelesen werden, die Christine de Pizan in ihrem »Livre des Trois Vertus« diesem Thema widmet. Demnach bestehe die Aufgabe der Dame vor allem darin, uber die Ehre ihrer Herrin zu wachen und die Liebe zwischen Fürst und Fürstin zu fördern, indem sie das Paar zu doulces paroles et amoureuses anhalte ${ }^{24}$. Madame de Parroy scheint ihre Aufgabe zur Zufriedenheit der Guise erledigt zu haben, denn später wurde sie zur Gouvernante der schottischen Königin ernannt, um die sie sich ebenfalls auf sehr zuverlässige Weise kummerte ${ }^{25}$. Neben der Ehrendame waren auch Annas neuer maître d'hôtel und ihr écuyer bereits ernannt und alle weiteren Posten ihres Haushaltes besetzt worden. Dem Vater hatte die Prinzessin geschrieben, ihre französischen Begleiter hätten Wert darauf gelegt, alle Italiener ohne Ausnahme nach Ferrara zurückzuschicken, und Giulio Alvarotti, Ercoles Gesandter in Frankreich, sollte kurze Zeit später von der Unmöglichkeit berichten, einige Worte mit der Prinzessin allein zu wechseln. Es scheint, als hätten die Guise die neue Herzogin von Aumale so schnell wie möglich in das französische Umfeld zu integrieren und, zumindest anfangs, ihren Kontakt zu aus Ferrara stammenden Personen einzuschränken gesucht. Anna selbst hatte sich während der Reise bis Grenoble um italienische und französische Begleiter und Begleiterinnen gleichermaßen bemüht, was nicht ohne Bewunderung zur Kenntnis genommen worden war:

Monsignor di Trois [Troyes] non lascia questa signora mai, et ella lo honora et accarezza, come anco trattiene tutti gli altri Francesi. Et in ciò mostra esser savia che havendo a vivere tra loro se gli mostri affittionata. Non è però che non facci amorevole dimostrationi alli nostri gentilhuomini e gentildonne, le quali tutte, dal hora che esce della camera fin che va a letto, non la abbandonano mail ${ }^{26}$.

${ }^{23}$ (Florimond) de Biencourt an François de Lorraine, 3. Nov. (1548), Moirans, in: BnF, Fr. 20553, fol. 7r.

${ }^{24}$ PIZAN, Livre des Trois Vertus, S. 90-120, Zitat S. 97. Vgl. die Beschreibung der Aufgabe der Ehrendame im Haushalt der Margarete von Österreich, 1525, in: Emmanuel de QUINSONAS, Matériaux pour servir à l'histoire de Marguerite d'Autriche, duchesse de Savoie, regente des Pays-Bas, Bd. 3, Paris 1860, S. 281: Madame aura une dame d'honneur, laquelle aura le regard sur la conduicte des filles d'honneur et autres femmes de son hostel.

${ }^{25}$ Antoinette de Bourbon an Marie de Lorraine, 4. Dez. 1553, in: Marie de LORRAINE, Queen of Scotland, Foreign Correspondence. From the originals in the Balcarres Papers, 1548-1557, hg. v. Marguerite WoOD, Edinburgh 1925, S. 183; Charles de Lorraine an dies., 8. Apr. 1557, Villers-Cotterêts, in: LORRAINE, Lettres, S. 261.

${ }^{26}$ Ferrante Trotti an Ercole d'Este, 14. Okt. 1548, Vercelli, in: ASM, Canc. duc., Amb. Francia 52. Beim Erzbischof von Troyes handelt es sich um Louis de Lorraine. Zum Folgenden: Louis de Lorraine an François de Lorraine, 25. Okt. (1548), Embrun, in: BnF, Fr. 20467, S. 70. 
Anfang November war für Anna der Zeitpunkt gekommen, die ferraresischen Edelleute zu entlassen. Die Zahl der Heimkehrenden war so groß, daß der Erzbischof von Troyes noch eine Woche zuvor seinem Bruder besorgt mitgeteilt hatte, es würde zwei Tage dauern, bis die gesamte Reisegruppe verabschiedet sei. In einem Schreiben an den Vater bedankte sich die Prinzessin für die Begleitung und lobte die guten Dienste, die ihr wăhrend der Fahrt geleistet worden waren:

Io non ho voluto manchar di basciar le mani alla E.V. della bona conpagnia che mi ha acconpagnato infino a Grenobles e supplicarla che mi vogli far questo favor, quando saranno arrivati a Ferrara, di fargli intender a tutti la sastifatione ch'io scrivo a V.E. d'haverne, perche certo s'io facessi altrimenti saria molto ingrata per la gran diligentia che hanno tutti usato verso $\mathrm{me}^{27}$.

Auch die Ferraresen waren zufrieden und begaben sich fort contens auf den Weg nach Italien, was jedoch, wie Biencourt mitteilte, ne fut sans bien pleurer. Die Entlassung ihres Gefolges bedeutete für die Prinzessin eine Trennung von Heimat und Herkunft, Sprache und Kultur, von ihrer Stadt und den Höfen ihrer Eltern, folglich war sie traurig und weinte, und trösten ließ sie sich nur von einem Brief ihres Gemahls: Anna, so ein Bericht an François, habe sich nach diesem Abschied zunächst toutte fachée et eplourée gezeigt, et soudaiment qu'elle reçeu de voz nouvelles nous recommansames à rire comme paravant ${ }^{28}$.

2. »A suono di pifferi« - Die Ankunft im Königreich

Der Triumphzug durch Frankreich und die Befürchtungen des Ferrareser Gesandten

Von Beginn an hatte der Schwager Louis der Prinzessin auf ihrer Reise Gesellschaft geleistet, und der Herzog von Aumale, der sich bei ihm für die treuen Dienste bedankte, erhielt eine enthusiastische Antwort: Il vous a pleu me remercier de la paynne que j'ay prinse. Je vous assure, Monsieur mon frere, que la painne que j'ay prins n'a pas esté grande, estant avec une si honneste princesse, que je pence qui n'en soit point au monde une plus accomplie de

${ }^{27}$ Anna d'Este an Ercole d'Este, 7. Nov. 1548, Lyon, in: ASM, Canc. duc., Cart. prin. est. $1568 \mathrm{~A} / 14$.

${ }^{28}$ (Florimond) de Biencourt und Louis de Lorraine an François de Lorraine, 3. Nov. (1548), Moirans, in: BnF, Fr. 20553, fol. 7v, Fr. 20467, S. 73. 
touttes vertu qu'elle ${ }^{29}$. Nun nahm sich Claude de Lorraine der Prinzessin an. Auch er verstand sich ausgezeichnet mit seiner Schwiegertochter, behandelte sie come fuse la Regina und verbrachte die meiste Zeit in ihrer Sănfte: Monsieur votre pere l'aime tant, qui ne l'avoit point à demy, et fault bien dire qui ly porte grant amytye $e^{30}$. Der Beobachter aus Ferrara, beeindruckt von der großen Zuneigung des Herzogs von Guise, erzählte von wertvollen Geschenken und Unterhaltungen, die Anna den langen Weg verkürzen sollten. Jeden Tag, so der Bericht, halte der Herzog eine neue Überraschung für die Prinzessin bereit, egal, ob sie durch Städte oder über Land führen: Non è posibile che padre voglia meglio a figliola ${ }^{31}$. Von Grenoble reiste die Prinzessin weiter nach Lyon. Der Herzog von Aumale, beunruhigt uber die Nachrichten von der schlechten Ausstattung seiner Braut und gemäß seinem Wunsch, daß sie sich bei ihrer Ankunft am Hofe en bon équipage befinden solle, hatte eine weitere Überraschung vorbereiten lassen: In Lyon erwarteten Händler die Prinzessin, bei denen sie sich alles aussuchen konnte, was ihr gefiel. Knapp zwei Wochen später hatte Anna die Trauer uber den Abschied von ihren italienischen Begleitern vergessen, und Louis konnte seinem Bruder mitteilen: Madame ma seure se porte autant bien comme vous et moy pourrions soubhaiter ${ }^{32}$.

Der Weg von Lyon nach Paris wurde zum Triumphzug. In Beaune, Dijon, Châtillon und Troyes wurden Freudenschüsse abgefeuert, Turniere veranstaltet und Bankette gegeben, und die Prinzessin wurde gleichermaßen von den Vertretern der Städte und den Edelleuten der Schlösser willkommen geheißen und, wie es scheint, ins Herz geschlossen: Sua E. è incontrata con qual magior honor che si faria alla maestà del re, e per tutti i loghi che pasamo Sua E. las [ci]a di sé grandissima afecione a nobilli et a gli altri ${ }^{33}$. Noch in Italien, $\mathrm{zu}$ Beginn ihrer Reise, hatte Anna damit begonnen, gemeinsam mit den Damen ihres Gefolges in großer Gesellschaft zu speisen. Aus Pavia, wenige Tage nach ihrer Abfahrt aus Mantua, hatte Ferrante Trotti berichtet, die Prinzessin

${ }^{29}$ Louis de Lorraine an François de Lorraine, 3. Nov. (1548), Moirans, in: BnF, Fr. 20467, S. 74.

${ }^{30}$ Germanico Savorgnano an Ercole d'Este, 20. Nov. 1548, Dijon, in: ASM, Canc. duc., Amb. Francia 52; (Florimond) de Biencourt an François de Lorraine, 3. Nov. (1548), Moirans, in: BnF, Fr. 20553, fol. 7v.

${ }^{31}$ Germanico Savorgnano an Ercole d'Este, 23. u. 27. Nov. 1548, Châtillon, Troyes, in: ASM, Canc. duc., Amb. Francia 52 u. OCCHIPINTI (Hg.), Carteggio, S. 207.

${ }^{32}$ Louis de Lorraine an François de Lorraine, 8. Nov. 1548, Lyon, in: BnF, Fr. 20467, S. 77. Vgl. Alfonso Gilioli an Ercole d'Este, 27. Okt. 1548, Gap, in: ASM, Canc. duc., Amb. Francia 52; Anna d'Este an dens., 1. (Nov. 1548), Grenoble, in: Ibid. Cart. prin. est. $1568 \mathrm{~A} / 14$. Dagegen berichtet Giulio Alvarotti in einem Brief an dens., 29. Nov. 1548, Nogent-sur-Seine, in: Ibid. Amb. Francia 45, uber die Aufnahme der Geschenke: Lei non volle nulla, dicendo che quando lo [François] conosceva lo obedirà poi in ogni cosa, ma al hora non voleva nulla e cosi non prese nulla.

${ }^{33}$ Germanico Savorgnano an Ercole d'Este, 23. Nov. 1548, Châtillon, in: ASM, Canc. duc., Amb. Francia 52. 
habe sich am Abend in ein Zimmer ihrer Herberge begeben, in das jeder eintreten durfte und wo der Tisch zum Essen gedeckt war. Dann habe sie eine ihrer Begleiterinnen rufen lassen, damit sie ihr Gesellschaft leiste, und sich bei den anderen entschuldigt, die sie wegen der Enge des Raumes nicht einladen konnte $^{34}$. Seit diesem Tag wurden die Damen an die Tafel der Prinzessin gerufen, ebenso diejenigen der Städte, in denen die Reisenden Halt machten, und bald gab es keinen Abend mehr, an dem nach dem Essen nicht getanzt und gefeiert wurde.

So waren es nicht nur die Anstrengungen des Weges, die dazu fuhrten, daß die Herzogin von Aumale gegen Ende ihrer langen Reise erschöpft war, auch die Lebensgewohnheiten des französischen Hofes, an denen sie Gefallen zu finden begann, leisteten ihren Beitrag. Anna, so ein Bericht an ihren Vater, come [sta] dui o tre giorni in riposo, fa miglior ciera, ma, stando in cammino, per il poco dormir più che per fatica, patisce alquanto, perché Sua Signoria illustrissima fa la usanza di questi principi di festegiar fin a megia notte $e$ levarsi a bonora. Ercoles Botschafter bei Hofe, der Ende November, wenige Tagesreisen von Paris entfernt auf die Reisegruppe getroffen war, fand die Prinzessin hingegen sana, bella e gratiossa al solito ${ }^{35}$. Sein an den Herzog von Ferrara gerichteter Brief, der für Ercole die erste unabhängige Schilderung darstellte, seit seine Männer die Tochter in Grenoble verlassen hatten, gab ein anderes Bild der Situation wieder als das, welches der bei den Empfängen und Turnieren, Festen und Banketten der Reise anwesende Savorgnano gezeichnet hatte. Sichtlich enttäuscht schrieb Alvarotti über sein erstes Treffen mit der Prinzessin, daß er kaum mit ihr habe sprechen können,

perché Monsignor di Guisa e Monsignor di Troes, che erano presenti, dissero: alla messa alla messa, cosi s'ando alla messa, e dalla messa alla menssa, e dalla menssa a montar ella in letica e li altri a cavalo, e con essa lei in letica Madama di Chiatovilon, di modo che in tutto hoggi io non le ho potutto dir' nulla senza testimonio. Smontati da cavalo ognuno ando ai suoi allogiamenti. Spogliato che io fui, andai da lei e trovai che Monsignor di Guisa e Monsignor di Troes la haveano in mezo, e la tenero cosi fin hora di una, che la mandono a cena [...]. E penso di doverla poco veder e meno parlarli in questo viaggio, e poi non so ciò che sarà in corte.

Und er fügt seiner Schilderung hinzu: V.E. mi dirà: Aspetta un poco che tu vedrai etc, et io Le dico che questo è stato un mal principio ${ }^{36}$. Das Bemühen

\footnotetext{
${ }^{34}$ Ferrante Trotti an Ercole d'Este, 14. Okt. 1548, Vercelli, in: ASM, Canc. duc., Amb. Francia 52.

${ }^{35}$ Germanico Savorgnano an Ercole d'Este, 12. Nov. 1548, Chalon-sur-Saône, in: OCCHIPINTI (Hg.), Carteggio, S. 204. Das Wort in Klammern wurde von mir anhand des Originals korrigiert. Giulio Alvarotti an Alfonso Rossetto, 3. Dez. 1548, Paris, in: ASM, Canc. duc., Amb. Francia 45.

${ }^{36}$ Giulio Alvarotti an Ercole d'Este, 29. Nov. 1548, Nogent-sur-Seine, in: ASM, Canc. duc., Amb. Francia 45. Vgl. den Brief vom 5. Dez. aus Paris, in: Ibid.: Io non potei quasi parlarli
} 
der Guise, die junge Frau von ferraresischem Einfluß fernzuhalten, erstreckte sich nicht nur auf die Auswahl von Franzosen und Französinnen für ihre maison, sondern auch auf ihre Isolierung von den Agenten des Vaters.

\section{"Come li fuse sorela«: Der Empfang in Paris und bei Hofe}

Derweil liefen in der Hauptstadt die Vorbereitungen für Annas Empfang. Bereits Anfang Oktober hatte sich der König erkundigt, wann die Prinzessin in Paris eintreffen würde und dem Herzog von Ferrara zugesichert, seine Tochter al paro di Madama sua suor behandeln zu wollen. Charles de Lorraine schrieb dem Bruder nach Bordeaux, Heinrich und der Hof erwarteten die Braut gespannt, und er selbst würde ihr entgegenreisen, sollte François nicht rechtzeitig vor ihrer Ankunft in Paris eintreffen. Nicht anders lauteten die Berichte, die Ercole aus Frankreich erhielt:

Io la posso bene assicurare che essa signora Principessa è aspettata e disiderata quanto maggiormente si può dal Re, dalla Reina, da madama [Margherita] e generalmente da tutta questa corte, e già Sua Maestà fa apprestare giostre, torniamenti et altre feste per onorar queste nozze più che altre nozze di principi alcuni che da un gran tempo in qua si facessero a questa corte 37 .

Anfang Dezember, zwei Tagesreisen von Paris entfernt, traf der Kardinal von Ferrara, begleitet von einer großen Anzahl italienischer Edelleute, auf seine Nichte. Zur gleichen Zeit waren François de Lorraine und Anne de Montmorency aus der Guyenne an den Hof zurückgekehrt, von wo aus sich der Herzog von Aumale sofort wieder auf den Weg machte, seiner Braut entgegenzureiten. Begleitet wurde er von seinen Brüdern und einer Truppe von uber hundert Reitern, und bereits am nächsten Tag, in Boissy-Saint-Léger, fanden sie die Prinzessin und ihr Gefolge. Anna und ihr Schwiegervater, vielleicht, um die Spannung dieses ersten Treffens etwas abzumildern, hatten sich dabei einen Scherz erlaubt. Eine der Damen, herrlich gekleidet und geschmückt, wurde François und seinen Männern als die neue Herzogin von Aumale vorgestellt und von diesen umarmt und geküßt. Erst als die burla entdeckt und ausgiebig belacht worden war, standen sich der Bräutigam und seine zukünftige Gemahlin zum ersten Mal gegenüber: Quanto fusse le careze

[...], quasi quando io havesse voluto dirle cosa che non havesse voluto essere inteso da loro. [...] Non le lassano mai parlar, massime da Italiani, che non vi siano essi alontorno. Bei Madame de Châteauvillain handelt es sich um Antoinette de La Baume-Montrevel.

${ }^{37}$ Giulio Alvarotti an Ercole d'Este, 9. Okt. 1548, in: ASM, Canc. duc., Amb. Francia 25; Charles de Lorraine an François de Lorraine, 18. Okt. 1548, Moulins, in: LORRAINE, Lettres, S. 115; Ippolito d'Este an Ercole d'Este, 29. Nov. 1548, Saint-Germain-en-Laye, in: OCCHIPINTI (Hg.), Carteggio, S. 208. 
et accoglienze non si può esprimere, ma molto più de tutti fu quella di Monsignor de [Aumale], a vedere una cosi belissima sposa. Die Beobachter waren sich uber den Erfolg dieses ersten Treffens einig: Non so che ciera si facessero insiemi la Signora prencipessa e Monsignor di Umala, schrieb Alvarotti dem Herzog von Ferrara, ma so bene che cavalcando insiemi a Parigi se la faceano buona $^{38}$. Gemeinsam setzten Anna und François die Reise fort. Antoinette de Bourbon gesellte sich zu ihnen, begleitet von zwei Töchtern des Grafen della Mirandola, die als Italienerinnen der Braut Gesellschaft leisten sollten. Die Kardinäle von Bourbon und Vendôme, die Herzöge von Nevers und Nemours sowie viele weitere Prinzen kamen ihnen entgegen, so daß Anna am Ende mit einer Begleitung von weit mehr als dreitausend Reitern die Tore von Paris erreichte. Die lange Reise der Prinzessin war zu Ende ${ }^{39}$.

Nachdem der Stadtrat von Heinrich aufgefordert worden war, Anna in Paris willkommen zu heißen und ihr alle nur mögliche Ehre zu erweisen, war beschlossen worden, die neue Herzogin von Aumale mit großem Pomp zu empfangen. In Begleitung der Räte, der Repräsentanten der Stadtviertel en grande compaignée, der Bogen- und Armbrustschützen und der Arkebusiere, in schwarzen Samt gekleidet und unter dem Donner der Kanonen, wollten der prévôt des marchands und die Ratsherren der Prinzessin bis zum Kloster Saint-Anthoine-des-Champs entgegengehen, allesamt Ehrbezeugungen, che questa vila non fa mai, senon al re, e lo fece anco allo imperatore quando passò per qua, wie Alvarotti seinem Herm versicherte. Doch auch ohne ausdrücklichen Befehl des Königs hätte es an einem respektvollen Empfang nicht gemangelt, war Anna doch Schwiegertochter des Herzogs von Guise, der von den Parisem seit seinem Sieg über die Engländer und der Niederschlagung des Bauernaufstandes in Lothringen als Beschützer der Stadt und Bewahrer der religiösen und sozialen Ordnung verehrt wurde ${ }^{40}$.

Am 4. Dezember 1548 war es soweit. Auf einem Grauschimmel, der eine goldbestickte und mit silbernen Fransen verzierte Schabracke aus schwarzem Samt trug, näherte sich die in überreiche Gold- und Silberbrokatstoffe gekleidete Prinzessin der Stadtmauer. Eine Viertelmeile bevor sie mit ihren Beglei-

38 Da Marano, Cronaca di Ferrara, fol. 12v; Giulio Alvarotti an Ercole d'Este, 5. Dez. 1548, Paris, in: ASM, Canc. duc., Amb. Francia 45. Der Bericht über die burla: Germanico Savorgnano an dens., 5. Dez. 1548, Paris, in: Ibid. Nr. 52.

${ }^{39}$ Michele della Torre an den Kardinal Famese, 18. Okt. 1548, Moulins, in: ROMIER, Origines, Bd. 1, S. 72f., Anm. 9. Alvarotti spricht von 3000 Reitern (Brief an Ercole d'Este vom 5. Dez.), das Protokoll des Stadtrats von 4000-5000 (4. Dez. 1548, in: Paul GúERN u.a. (Hg.), Registres des délibérations du Bureau de la ville de Paris, Bd. 3, Paris 1886, S. 140).

${ }^{40}$ Entscheidungen des Stadtrats, 26. Nov. 1548, in: GuÉRIN u.a. (Hg.), Registres des délibérations, Bd. 3, S. 139; Giulio Alvarotti an Ercole d'Este, 5. Dez. 1548, Paris, in: ASM, Canc: duc., Amb. Francia 45. Eine detaillierte Beschreibung von Annas Empfang und ihren ersten Tagen bei Hofe auch in: Georgio Conegrani an Francesco Gonzaga, 7. Dez. 1548, Poissy, in: ASMan, AG 641, fol. 275-278. 
tern das Tor erreichte, präsentierten sich die Abgeordneten der Stadt, und nachdem die ersten Freudenschüsse abgefeuert worden waren, setzte der prévôt des marchands zu einer Rede an, aus der das Verhältnis zwischen der Stadt Paris, König Heinrich, der Familie der Guise und der zukünftigen Herzogin von Aumale zu ersehen ist:

Combien, Madame, que les habitans de Paris, ville cappitalle du Royaulme, n'ayent acoustumé d'aller au devant, sinon du Roy, leur Prince et souverain seigneur, et de la Royne, à sa premiere entrée en la Ville, toutesfoys [...], nous vous venons au devant faire la reverance et porter l'honneur que nous sommes tenuz et qui vous appartient, pour estre princesse yssue de la couronne et maison de France, acomplye de plusieurs graces et dons de nature, et douée de très grandes et très excelentes vertuz. Aussi, Madame, pour l'aliance que vous venez prandre en la maison de Monseigneur le duc de Guyse, prince duquel la Ville a receu tant de graces, de faveurs et biensfaictz, et pareillement de Messeigneurs le Reverandissime cardinal et duc d'Aumalle, ses enffans, que les habitans d'icelle leur en seront perpetuellement tenuz et obligez, et à ceulx qui leur attiennent ${ }^{41}$.

Ihre königliche Herkunft und der Wert der Familie ihres zukünftigen Gemahls, aber auch die eigenen graces, dons und vertuz sollten von Beginn ihres Lebens in Frankreich an Stand und Ansehen der jungen Frau bestimmen. Anna, die sich der Bedeutung der französischen Hauptstadt firr die Geschicke der Familie der Guise und fur ihre eigene Zukunft bewußt gewesen zu sein scheint, bedankte sich bescheiden furr die ihr erwiesene große Ehre, beaucoup plus grand qu'il ne m'appartient, und versprach den Parisern, sich immer und überall de très bon cueur furr die Stadt einsetzen zu wollen. Diese Worte, so der Chronist, sprach die Prinzessin con tanta grazia e tanta benignitate, che ciascuno restò più che sodisfato e pieni di maraviglia ${ }^{42}$.

Unter dem Donner weiterer Kanonenschüsse und mit den Kardinälen von Bourbon und von Ferrara an ihrer Seite zog Anna schließlich in die Hauptstadt ein. Mit rund 300000 Einwohnern war Paris die bevölkerungsreichste Stadt Europas, in ihren Straßen und Gassen drängten sich schon an gewöhnlichen Tagen Fußgänger, Reiter und Karren, so daß der Stadtrat für den Empfang der Prinzessin Bewaffnete bereithielt, um die Menschenmenge unter Kontrolle zu halten ${ }^{43}$. Annas Triumphzug endete am Hôtel de Reims nahe der heutigen Place Saint-Michel, wo sie von Antoinette de Bourbon a suono di pifari empfangen wurde. Antoinette hatte die Schwiegertochter zwar schon vor den To-

${ }^{41}$ Protokoll des Stadtrats, 4. Dez. 1548, in: GUÉRIN u.a. (Hg.), Registres des délibérations, Bd. 3, S. $139 f$.

${ }^{42}$ Protokoll des Stadtrats, 4. Dez. 1548, in: GuÉRN u.a. (Hg.), Registres des délibérations, Bd. 3, S. 140; DA MARANO, Cronaca di Ferrara, fol. 13r.

${ }^{43}$ Protokoll des Stadtrats, 26. Nov. 1548, in: GUÉRIN u.a. (Hg.), Registres des délibérations, Bd. 3, S. 139. Zur Stadt Paris: Jean-Pierre BABelON, Paris au XVI siècle, Paris 1986. Die Schätzung der Einwohnerzahl: Jean JACQUART, Paris: First Metropolis of the Early Modern Period, in: Peter ClaRK, Bernard LEPETIT (Hg.), Capital Cities and their Hinterlands in Early Modern Europe, Aldershot, Brookfield 1996, S. 105-118, hier S. 105. 
ren der Stadt begrußt, war dann aber vorausgeeilt, um ihre Ankunft in Paris vorzubereiten. Auch Heinrich hatte sich in die Hauptstadt begeben, per far' un par' di festive secretamente, und am Abend stattete er der Herzogin von Aumale einen Besuch $\mathrm{ab}^{44}$. Als Anna von der Ankunft des Königs erfuhr, ging sie ihm entgegen, verbeugte sich und wollte dem Herrscher den Mantelsaum küssen,

ma Sua Maestà gli volse baciare la facia. E cosi entrerno in camera et anderno a sedere sopra due sedie e ragionavano loro dui. E doppo certe poche parole Sua Maestà gli disse che havea sempre desiderato vederla, e doppo che il Signor Iddio gli havea concesso de haverla nel suo regno che lui non l'accetava per cugina ma si per carnale sorella, e che mai Sua Maestà non si vederebbe satio di farli sempre cosa che li fosse in piacere, e molte altre parole fiono usate tra Sua Maestà e la Signora prencipesa ${ }^{45}$.

Tatsächlich behandelte Heinrich die Prinzessin von Anfang an, come li fuse sorela. Sie speisten gemeinsam mit den Herzoginnen von Valentinois und von Guise, und der König tanzte mit der jungen Frau. In seinem diese erste Begegnung erwähnenden Brief an den Herzog von Ferrara ließ er es an Hinweisen auf die gemeinsame Herkunft und das königliche Blut, welches in Annas Adern flösse, nicht fehlen:

J'ay esté faire ung tour à Paris, saichant que ma cousine la princesse votre fille y devoit arriver, laquelle j'avoys la plus grande envye de veoir qu'il estoit possible. Et depuis l'ayant veue et congneu ses vertuz, avec la semblance qu'elle porte au visage du lieu dont elle et moy sommes yssuz, $j$ 'ay sa présence tant agreable, qu'il me semble que je ne la puis assez veoir ${ }^{46}$.

Wie eine Schwester wurde Anna auch von der Königin und den anderen Damen des Hofes empfangen, gemeinsam verbrachten sie die folgenden Tage bei der Jagd und bei Turnieren, die Nächte bei Banketten und Bällen, und die Poeten widmeten ihre Werke $A$ tresnoble et tresflorissante Princesse Mme la Princesse de Ferrare, du temps de sa venue en France, et de ses nopces avec $M$. d'Aumale. Einer der Berichte vom französischen Hof jener Tage lautete: Tutta la corte sta in festa et allegrezza, come se lo Imperatore fusse di là da l'India. In somma di qua si vive alla carlona [sorglos] senza pensar hoggi quel

44 Giulio Alvarotti an Ercole d'Este, 5. Dez. 1548, Paris, in: ASM, Canc. duc., Amb. Francia 45; Michele della Torre an den Kardinal Farnese, 30. Nov. 1548, Poissy, in: Correspondance des nonces en France Dandino, della Torre et Trivultio (1546-1551), avec des documents relatifs à la rupture des relations diplomatiques 1551-1552, hg. v. Jean LESTOCQUOY, Rom, Paris 1966, S. 416.

${ }^{45}$ DA MARANO, Cronaca di Ferrara, fol. 13v. Das folgende Zitat: Germanico Savorgnano an Ercole d'Este, 5. Dez. 1548, Paris, in: ASM, Canc. duc., Amb. Francia 52.

${ }^{46}$ Heinrich II. an Ercole d'Este, 16. Dez. 1548, Saint-Germain-en-Laye, in: ASM, Canc. duc., Cart. prin. est. 1559 A/2. 
che possi essere domani, während es uber die Prinzessin aus Ferrara hieß, es könne ihr nicht besser gehen und sie würde von allen verwöhnt ${ }^{47}$.

\section{Hochzeit und Geschenke}

Am 16. Dezember 1548, einem Sonntag, wurde in Saint-Germain-en-Laye die Hochzeit gefeiert. Der König und die Königin, der ganze Hof sowie die Gesandten der größten Fürsten Europas nahmen an der Messe und dem anschlieBenden Festbankett teil. Die Braut war gekleidet in abito di regina, über ihrem prächtigen Kleid trug sie einen mit Hermelin besetzten Samtmantel, der in einer fast drei Meter langen Schleppe endete. Ihren Hals schmückte ein Diamantkollier, ihr Haar eine Haube aus Diamanten und Rubinen, quali valeano un gran tesoro. Auf der Stim trug sie einen Rubin, auf dem Kopf, come regi$n a$, eine Krone. Vom König und von ihrem zukünftigen Gemahl wurde Anna in ihren Gemächern abgeholt und a suon di pifari in die herrlich geschmückte Schloßkirche geleitet. Der Kardinal von Guise, unterstutzt von seinem Kollegen aus Ferrara, leitete die Zeremonie, und als con uno ellegantissimo sermone der Heiratsvertrag gelesen und die Messe zelebriert worden war, war aus der Prinzessin von Ferrara die Herzogin von Aumale geworden ${ }^{48}$.

Gültigkeit konnte die Ehe jedoch erst beanspruchen, wenn sie vollzogen war, und so wurde die Braut, nachdem den ganzen Tag und die halbe Nacht alla gagliarda und alla alemana getanzt worden war, vom König und der Königin am Arm genommen und in einen prächtig geschmückten Raum geführt, dessen Wände mit grünem Samt und Goldbrokat bespannt waren. Hier stand ein Bett mit einer Decke, die mit Edelsteinen und Perlen bestickt war: $E$ poi vene in camera Monsignor de Umala, suo sposo, con alcuni suoi compagni, et stetero in dolci ragionamenti circa un' ora. E Sua Maestà e la regina si partirno, e li sposi furno messi a letto ${ }^{49}$. Zwei Wochen lang wurden Bälle und Bankette, Maskeraden und Turniere veranstaltet, die das Königreich ein Vermögen kosteten. Am letzten Tag der Hochzeitsfeierlichkeiten schrieb der

\footnotetext{
${ }^{47}$ Montemerlo, Sekretär des Nuntius, an den Kardinal von Santa Fiora, 12. Dez. 1548, Lyon, in: Correspondance Trivultio, S. 417; Germanico Savorgnano an Ercole d'Este, 5. Dez. 1548, Paris, in: ASM, Canc. duc., Amb. Francia 52. Die Widmung der »Ruisseaux" von Charles Fontaine, Lyon 1555, in: Marie Madeleine FONTAINE, Dédicaces lyonnaises aus Guises-Lorraines, in: Yvonne BELLENGER (Hg.), Le mécénat et l'influence des Guise. Actes du Colloque de Joinville 1994, Paris 1997, S. 39-79, hier S. 70f.

${ }^{48}$ Giulio Alvarotti an Ercole d'Este, 16. Dez. 1548, Poissy, in: OCCHIPINTI (Hg.), Carteggio, S. 209; DA MARANO, Cronaca di Ferrara, fol. 16r. Vgl. den für Karl V. verfaßten Bericht "Bodas de Monseñor de Omale«, o.D. (Anfang 1549), in: AN, $21 \mathrm{Mi} 92$, und die Anklindigung einer "Descrittione delle giostre et feste fatte nelle nozze di Monsignor illustrissimo di Omalla con la Signora prencipessa di Ferrara « für den Herzog von Mantua, die selbst aber fehlt, in: ASMan, AG 642, fol. 18r.

${ }^{49}$ Da Marano, Cronaca di Ferrara, fol. 17r.
} 
ferraresische Gesandte, für banchetti, vestir', mascarate et spese di giostre seien uber 100000 Scudi ausgegeben worden: Non è ricordo di persona d'una tanta spesa e d'un tanto e cosi longo trionpho come è stato questo per questa causa $^{50}$. Allein der Wert der kostbaren Geschenke, mit denen die Braut regelrecht überschüttet wurde, belief sich auf unschätzbare Summen. Zwar hatte sich Alvarotti nach der Ankunft Annas bei Hofe noch besorgt uber die geringe Freigebigkeit des Königs gezeigt, doch am Morgen nach der Hochzeit besuchte Heinrich gemeinsam mit der Königin die Neuvermählte und überreichte Edelsteine und kostbare Stoffe. Von ihrem Gemahl erhielt die Prinzessin drapi d'oro, aneli et altre cose, und weitere Geschenke wurden ihr von den Angehörigen des Hofes gemacht:

Monsignor di Ghisa mandò a donare alla prencipessa tante collane e cinture e medaglie $e$ perle e molte burdure di più sorte [...] et altre diverse cose da don[n] e, che furno estimate di valore de 4000 scudi. Et el $r .^{\text {mo }}$ cardinale de Ghisa gli mandò a donare un colaro de diamanti, fatto con delli rubini et smeraldi, ch'era di valore di 2000 scudi. E la Regina le mandò a donare molte pezze de pano d'oro e d'argento di valore de 2000 scudi, et il $R^{m o}$ cardinale di Lorena le donò un letto fornito con la coperta di damasco cremesina, tutta recamata a forame d'oro, con una travata fatta similmente. Et anco li mando molti altri signori e baroni a donare cose belissime e di gran valore 51 .

Neben weiteren Edelsteinen und Perlen erhielt Anna goldenes Geschirr, ein kunstvoll gearbeitetes Schachbrett mit goldenen Figuren, ein silbernes Hündchen, in dessen Körpermitte sich eine Uhr befand, mehrere prachtvolle Betten mit den dazugehörigen Baldachinen, Überzligen und Paradekissen sowie ein Reitpferd nebst kostbarer Schabracke, Sattel und Zaumzeug ${ }^{52}$.

Das große Interesse des Herzogs von Ferrara am Wert der Geschenke, die seine Tochter von den Mitgliedern ihrer neuen Familie erhielt, rührte sicher zu einem großen Teil von der Tatsache her, daß die Guise laut Heiratsvertrag dazu verpflichtet waren, Anna ihrem Rang entsprechend mit Schmuck auszustatten. Die teilweise befremdlich wirkenden Schätzungen von Summen, in den Briefen der Berichterstatter des Herzogs immer dann zu finden, wenn jene auf die Geschenke zu sprechen kommen, ist daher nicht allein auf ein verständliches $\mathrm{Ma} B$ an Neugier seitens der Brauteltern zurlickzuführen, sondern auch auf ein rein geschäftliches Interesse des Herzogs an der Einhaltung eines

${ }^{50}$ Giulio Alvarotti an Ercole d'Este, 30. Dez. 1548, Poissy, in: ASM, Canc. duc., Amb. Francia 25. DA MARANO, Cronaca di Ferrara, fol. 18r, schätzt die Ausgaben des Königs auf 120000 Scudi, ohne jedoch die Geschenke für die Braut, das Turnier und die neue Kleidung mitzurechnen.

${ }^{51}$ DA MARANO, Cronaca di Ferrara, fol. 17r-v. Vgl. Giulio Alvarotti an Ercole d'Este, 6. u. 9. Dez. 1548, Paris, Poissy, in: OCCHIPINTI (Hg.), Carteggio, S. 208 u. ASM, Canc. duc., Amb. Francia 25.

${ }^{52}$ ESTE, Inventaire des meubles; DA MARANO, Cronaca di Ferrara, fol. 18vf.; Giulio Alvarotti an Ercole d'Este, 14. Nov. 1548, 6. Jan. 1549, in: OCCHIPINTI (Hg.), Carteggio, S. 205, 209 f. 
Vertrages. Darüber hinaus stellt die Übergabe eines Geschenkes eine soziale Transaktion dar, die das Verhältnis von Schenker und Beschenktem versinnbildlicht sowie Stellung und Ansehen der Akteure definiert. Die Berichte seiner Gesandten stellten für Ercole daher einen verlaßlichen Gradmesser für die Anerkennung seiner Tochter und mithin seines Hauses am französischen Hof dar ${ }^{53}$.

\section{Formalitäten zur Integration einer Ausländerin} Ratifikation des Ehevertrags und lettres de naturalité

Was nun noch fehlte, war die Ratifizierung des Heiratsvertrages und die Auszahlung der Mitgift durch die königlichen Kassen. Eine Woche nach der prachtvollen Hochzeitszeremonie in der Schloßkirche von Saint-Germain hatte Heinrich der Prinzessin und ihrem Gemahl eine jährliche Rente von 10000 Livres zugestanden, die als Teil der Mitgift dazu gedacht war, die Schulden der Krone beim Herzog von Ferrara zu begleichen ${ }^{54}$. Obwohl François in den folgenden Wochen mit weiteren lukrativen Ämtern versehen wurde und der König dadurch seinen guten Willen offenbarte, war das Schriftstück über die jährliche Rentenzahlung im Februar noch immer nicht vom Pariser Parlament ratifiziert, daher vorlăufig wirkungslos, und wurde schließlich nur auf ausdrücklichen Befehl Heinrichs registriert. Erst jetzt konnte die Ratifizierung des Heiratsvertrages auch durch die Guise vorgenommen werden, ein Schritt, der von Ercole ungeduldig erwartet wurde. Ende Mai 1549 waren die Vorbereitungen soweit abgeschlossen, daß Alvarotti berichten konnte, der Vertrag befände sich in ottima forma ${ }^{55}$.

Unterzeichnet wurden die Ratifizierung sowie die vorgesehene Schenkung des Herzogtums Guise in den folgenden Wochen, so daß für beide Seiten nur noch die Ausstellung verschiedener Empfangsbestätigungen blieb: Die Guise mußten dem Herzog von Ferrara den Erhalt der Mitgift quittieren, und Ercole konnte dem König die Tilgung seiner Schulden bestätigen. Doch auch dieser Vorgang zog sich in die Länge. Obwohl Ercole seinem Botschafter noch im Sommer Entwürfe für die Quittungstexte geschickt hatte, sollte es erst zu

${ }^{53}$ Vgl. Gadi AlgazI, Doing Things with Gifts, in: Ders. u.a. (Hg.), Negotiating the Gift. Pre-Modern Figurations of Exchange, Göttingen 2003, S. 9-27.

${ }^{54}$ Marie-Noelle BaUdouIN-MATUSzEK, Anne MERLIN-Chazelas (Hg.), Catalogue des actes de Henri II, Bd. 2, Paris 1986, S. 457. Das Dokument wurde von Heinrich II. am 23. Dez. 1548 in Saint-Germain-en-Laye unterzeichnet.

${ }^{55}$ Giulio Alvarotti an Ercole d'Este, 23. Jan. u. 30. Mai 1549, Poissy, in: ASM, Canc. duc., Amb. Francia 26, 45. Zur Registrierung des Vertrags beim Parlament, 4. März 1549: (Mathurin) Richer an François de Lorraine, 8. Febr. (1549), Paris, in: BnF, Fr. 20469, S. 127. Die Ratifizierung wurde am 4. Juni 1549 von den Notaren und einen Tag später von Claude und François de Lorraine sowie Antoinette de Bourbon unterzeichnet, in: ASM, Casa 358. 
Beginn des folgenden Jahres zur endgültigen Freisprechung des Brautvaters von jeglicher hinsichtlich der Mitgift eventuell noch bestehender Schuld kommen. Erst jetzt, lange nach der Unterzeichnung des Heiratsvertrages in Ferrara, der Hochzeitsfeier in Saint-Germain und nachdem Anna bereits ihr erstes Kind zur Welt gebracht hatte, waren die Formalitäten um ihren Ehevertrag und die Zahlungen ihres jährlichen Einkommens auch formal geregelt ${ }^{56}$.

Den letzten Schritt für Annas Integration in die französische Gesellschaft stellte die Ausfertigung ihrer lettres de naturalité durch den König dar. Ne voullant qu'elle tumbe à la discretion des ordonnances et constitutions généralles de notre royaume, befreite Heinrich mittels dieses Schriftstückes die Herzogin von Aumale vom Status der Ausländerin und ermöglichte ihr somit, Verträge zu schließen, Käufe, Verkäufe und Schenkungen zu tätigen, ihren Besitz nach Gutdüken zu verwalten und mit ihrem Eigentum so zu verfahren, wie es ihr richtig erschiene, ganz so, als sei sie im Konigreich geboren. Dies bedeutete in erster Linie, daß Annas gegenwärtiges und zukünttiges Eigentum dem droit d'aubaine entzogen wurde, es im Falle ihres Todes also nicht von den Beamten des Königs konfisziert werden konnte, wie es mit Erbschaften nicht naturalisierter Ausländer in der Regel geschah. Die lettres de naturalité der Herzogin von Aumale erlaubten daher explizit, que les enffans aussy qu'elle aura et autres ausquelz elle pourra disposer de sesdits biens, luy puissent, après son trespas, succedder. Mit diesem Schriftstïck war Anna auch rechtlich zur Französin geworden ${ }^{57}$.

3. »Francese visu, verbo et opera« - Eine Italienerin bei Hofe

Renées älteste Tochter war nun Herzogin von Aumale, Gemahlin eines der angesehensten Männer des Hofes und Angehörige einer der mächtigsten Familien des Königreiches. Bevor sie Anfang Dezember zum ersten Mal zusammengetroffen waren, hatte François seine Braut nur von den Portrăts Girolamos da Carpi und von den Beschreibungen derer gekannt, die der Prinzessin auf ihrer Reise Gesellschaft geleistet hatten oder ihr unterwegs begegnet waren. Als une des plus belles et honnestes princesses que j'aye jamais veu sowie

\footnotetext{
${ }^{56}$ Quittungen vom 20. Jan. u. 5. Febr. 1550, in: ASM, Casa 358. Zu den Problemen bei der Auszahlung der Mitgift: Caso in italiano in materia delli 150 mila Franchi di Monsignor di Umala, o.D. (Sommer 1549), in: Ibid.; Ercole d'Este an Giulio Alvarotti, 28. Juli 1549, in: ASM, Canc. duc., Amb. Francia 39.

${ }^{57}$ Lettres de naturalité fur Anna d'Este, ausgestellt durch Heinrich II. in Saint-Germain-enLaye, ratifiziert durch den Rechnungshof am 13. Dez. 1548, in: AST (Cast.), Int., PGN, cat. 2, m. 10, n. 1 .
} 
als autant belle, sage et vertueuse qu'il y en ayt point au monde war Anna ihrem zukünftigen Gemahl geschildert worden, und Biencourt hatte versichert: C'est la plus doué et humaine princesse qu'il est posible de voir. Die burla, die sich anläßlich des ersten Zusammentreffens Claude und seine Schwiegertochter mit dem Herzog von Aumale erlaubt hatten, zeigt die Austauschbarkeit der jungen Frau zu diesem Zeitpunkt. François kannte die Prinzessin nur durch die Beschreibungen Dritter, folglich konnte er nicht anders, als in dem festlich gekleideten Mädchen, das ihm als Tochter des Herzogs von Ferrara präsentiert wurde, seine kluge, tugendhafte und wunderschöne Braut $\mathrm{zu}$ erkennen ${ }^{58}$.

\section{Anna d'Este und François de Lorraine}

Von der >echten` Anna zeigten sich die Guise keineswegs enttăuscht, scheint sie doch ihre hohen Erwartungen nicht nur erfüllt, sondern gar ubertroffen zu haben. Die Vorstellungen, welche sich der Herzog von Aumale vor ihrem ersten Treffen von seiner Braut und dem künftigen Eheleben gemacht hatte, sind aus einem Brief an seine Schwester Marie abzulesen. Noch keine achtzehn Jahre sei die Prinzessin aus Ferrara alt und dennoch aussy grande que vous, schreibt François nach Schottland und berichtet von Briefen, die er vom Vater erhalten habe, nachdem dieser in Grenoble mit seiner künftigen Gemahlin zusammengetroffen sei, und deren Schilderung ihn von Ferne verliebt gemacht habe. Madame, les ungs se marient pour apres se reposer en leur mayzons mes [mais] je ne suis de ceulx la, umreißt der Herzog daraufhin sein künftiges Leben, das auch weiterhin vom Dienst für den König bestimmt sein würde, während er sich von seiner Gemahlin möglichst bald einen Sohn erhoffte $e^{59}$.

Rund zehn Tage nach dem ersten Treffen des Paares erfuhr Ercole von der großen Zufriedenheit des Bräutigams, und die Begeisterung, die aus dem Brief von Antoinette de Bourbon spricht, ihr Hinweis auf le grand plaisir et contentement que j'ay reçeu de sa présence sowie das Lob für die gute Erziehung seiner Tochter werden dem Herzog zusätzlich geschmeichelt haben: Ayant, Monsieur, retrouvé en elle ce que $j$ 'ay tousiours esperé de votre nourriture, et

\footnotetext{
${ }^{58}$ Vassé und Maugiron an François de Lorraine, 18. u. 22. Okt. 1548, in: POYTIERS, Lettres, S. 31, Anm. 4; (Florimond) de Biencourt an dens., 3. Nov. 1548, Moirans, in: BnF, Fr. 20553, fol. 7r. Vgl. François d'Orléans an Marie de Lorraine, 9. Dez. 1548, SaintGermain-en-Laye, in: Marie de LORRAINE, Queen of Scotland, Foreign Correspondence. From the originals in the Balcarres Papers, 1548-1557, hg. v. Marguerite WoOD, Edinburgh 1925, S. 20: Anna sei une des belles et honneste princesse que l'on souroit voir.

${ }^{59}$ François de Lorraine an Marie de Lorraine, 9. Nov. 1548, Bordeaux, in: LORRAINE, Balcarres, S. 19.
} 
que l'on pourroit souhaitter pour le myeulx en toute perfection ${ }^{60}$. Doch auch Anna selbst wird mit einer bestimmten Vorstellung von ihrem Brăutigam und dem Leben, das sie in Zukunft fuhren würde, an den Hof gekommen sein. Wahrend ihrer Reise hatte die Prinzessin viel mit ihren französischen Begleitern über ihren Gemahl gesprochen, dem schon im Vorhinein berichtet worden war, que jamais princesse ne porta plus d'honeur et amytyé à mari qu'elle vous fera. ${ }^{61}$ Und sicher wird sie die Frage nach Wesen und Aussehen des Herzogs von Aumale gestellt haben. Als ernst, aber gütig und leutselig, ohne Hochmut, und mit sehr charakteristischem Äußeren war François von einem ferraresischen Beobachter beschrieben worden:

Era huomo cosi de 35 anni [tatsăchlich aber 29], grande di persona e bene proportionato, con la facia alquanto longhetta, con occhi biascchi [?], il naso aquilino, stortetto per una ferita de una lanza che gli era stata data giostrando, e la sua aira havea più presto del sacro che del' aliegro. Ma però era huomo molto affabile e benigno, nel parlare senza alcuna altareza, considerando el gran stato dove el si ritrovava et il grado che lui tiene apreso Sua Maestà, et anco essendo huomo valentissimo nelle armi ${ }^{62}$.

Die Tatsache, daß sich der Chronist in der Schätzung des Alters irrt, unterstreicht noch die Autorität, die von François ausging. Auch Jahre später zeigten sich die Berichterstatter stets beeindruckt von seinem ausgezeichneten Ruf bei Hofe, wurde der Herzog doch furr seine cortesia et bon governo von allen Seiten gelobt. In ihrem ersten Brief nach der Hochzeit schrieb Anna dem Vater, sie habe all das bestatigt gefunden, was dieser ihr vor ihrer Abreise aus Ferrara über François erzählt habe, und sie bedankte sich bei Ercole für die

sattisfatione grande e contento ch'io ho di haver havuto un tal principe come Monsignor d'Omala, dil che ne ringratiarò sempre humilissimamente V.E. di havermi cosi ben messa. E gli prometto che ho trovato vero tutto quello che mi diceva la $E$.V., mentre che io ero a Ferrara, di lui, tanto che saressimo contenti tutti dui ${ }^{63}$.

Tatsăchlich war die junge Herzogin von Aumale glücklich. La S. ${ }^{r a}$ Prencipessa sta tutta contenta ed amata infinitamente da mons.' di Umala et ella altretanto ama lui, erfuhr Ercole Ende Dezember, und im Januar berichtete Alva-

${ }^{60}$ Giulio Alvarotti an Ercole d'Este, 15. Dez. 1548, Poissy, in: ASM, Canc. duc., Amb. Francia 45; Antoinette de Bourbon an dens., 16. Dez. 1548, Saint-Germain-en-Laye, in: Ibid. Cart. prin. est. $1568 / 13$.

${ }^{61}$ (Florimond) de Biencourt an François de Lorraine, 25. Okt. (1548), Embrun, in: BnF, Fr. 20555, fol. 153r.

${ }^{62}$ DA MARANO, Cronaca di Ferrara, fol. 8r. Tatsächlich hatte François die Narbe während der Belagerung von Boulogne 1545 erhalten. Zum Folgenden: Ercole Strozzi an Guglielmo Gonzaga, 17. Febr. 1553, Paris, in: ASMan, AG 645; Giovanni Michiel, Relazione fur die Serenissima, 1561, in: TOMMASEO (Hg.), Relations, Bd. 1, S. 440.

${ }^{63}$ Anna d'Este an Ercole d'Este, 29. Dez. 1548, Saint-Germain-en-Laye, in: ASM, Canc. duc., Cart. prin. est. 1568 A/14. 
rotti, sie sei nicht nur zufrieden mit ihrem Gemahl und dessen Familie, sondern auch mit dem König, der Königin und dem gesamten Hof. Noch zehn Jahre später sollte aus Frankreich zu erfahren sein, daß Anna sich mit ihrem Gatten sowie den Herren und Damen des Hofes ausgezeichnet verstehe ${ }^{64}$.

Nach den ersten gemeinsam verbrachten Monaten scheint sich die gegenseitige Zuneigung noch vergrößert zu haben, und insbesondere François zeigte sich so verliebt, wie es die Damen des Hofes kaum für möglich gehalten hătten: Madama d'Aumala sta benissimo et ogni dì è più contenta. Il Signor suo marito mostra in modo di amarla che le donne della corte dicano che non ha altro fine al mondo che di compiacerla ${ }^{65}$. Auch die Schwiegermutter fand den ersten Eindruck, den Anna hinterlassen hatte, bestätigt und war glücklich, für ihren Sohn die richtige Frau gefunden zu haben:

Si à son nouvel advenement elle nous a donné toutes occasions de luy faire le traitement qui luy est deu, elle augmente en nous chacun jour la bonne volunté et singuliere affection que nous avons de luy continnuer. Sur quoy, Monsieur, je vous diray que je me repute et estime très heureuse que mon filz aye trouvé [...] une si honneste et vertueuse princesse ${ }^{66}$.

Michel de L'Hospital, der anläßlich der Vertragsunterzeichnung in Ferrara als Kurator der jungen Frau aufgetreten und mit ihr dann gemeinsam nach Frankreich gereist war, hatte sie in einer Epistel auf ihre neuen Aufgaben vorzubereiten versucht. Es müsse nun ihr oberstes Ziel sein, dem Gemahl zu gefallen, und solange sie noch keine Kinder habe, solle Anna die Studien ihrer Jugendzeit fortsetzen, lautete sein Rat. Der an den Lärm des Schlachtfeldes gewöhnte Herzog wüßte es nämlich zu schätzen, im Gespräch mit einer gebildeten Gefärtin den notwendigen Ausgleich zu finden ${ }^{67}$. Die Vorstellung, Anna und François würden sich gegenseitig ergänzen, kommt auch in einem Gedicht des Giovan Battista Giraldi Cinzio zum Ausdruck, in dem das Verhältnis des Herzogspaares als das eines kostbaren Schmuckstückes besungen wird. Der Edelstein werde durch seine goldene Fassung schöner, während das Gold der Fassung durch den Stein an Wert gewinne: Che quanto, oltre che fia leggia-

64 Giulio Alvarotti an Ercole d'Este, 30. (nicht 6.1) Dez. 1548, 21. Jan. 1549, Poissy, in: FonTANA, Renata di Francia, Bd. 2, S. 291 u. ASM, Canc. duc., Amb. Francia 26; Ders. an Alfonso d'Este, 24. Aug. 1560, in: Ibid. Nr. 36, fasc. II, fol. 62v.

${ }_{65}$ Ferrante Trotti an Ercole d'Este, 10. März 1549, Saint-Germain-en-Laye, in: ASM, Canc. duc., Amb. Francia 52. Vgl. den Hinweis auf [le] devoir de bon mari que faictes à ce commancement de mesnage in einem Brief von Antoine de Bourbon an François de Lorraine, 25. Mărz (1549), Pau, in: Antoine de BOURBON, Jehanne d'AlBreT, Lettres, hg. v. Achille de RochambeaU, Paris 1877, S. 14.

${ }^{66}$ Antoinette de Bourbon an Ercole d'Este, 10. Mai 1549, Saint-Germain-en-Laye, in: ASM, Canc. duc., Cart. prin. est. 1568/13.

${ }^{67}$ DuPRÉ LASALE, L'Hospital, S. $132 \mathrm{f}$. 
dra, e bella, / Anna havrà honor dal suo marito egregio, / Tanto darà al consorte anch'ella pregio ${ }^{68}$.

Der Herzog von Ferrara konnte mit seiner Wahl zufrieden sein und erhielt Glückwünsche von denjenigen, die den Empfang seiner Tochter in Frankreich miterlebt und das Ansehen, welches die Guise, vor allem aber sein Schwiegersohn François genossen, voll Bewunderung zur Kenntnis genommen hatten:

Io, come minimo servitor di V.S.I., mi alegro infinitamente quanto la E.V. abbia colocata bene la ill. ${ }^{\text {ma }}$ sua figliola, che dopoi che giongiemo nel regnio di Sua Maestà si a veduto tanta estimacione di questa ill. ${ }^{\text {ma }}$ fameglia di Guisa e particularmente del ill. ${ }^{\text {mo }}$ Signor duca d'Umala, ch'io né gli altri l'avevamo mai creduto'.

Und Ercole, nachdem er die Berichte uber die herzliche Aufnahme der Prinzessin bei Hofe, über die außergewöhnlichen Hochzeitsfeiern und reichen Geschenke erhalten hatte, schrieb einen Dankesbrief an seinen Bruder, denn schließlich war es der Kardinal gewesen, der das französische Heiratsprojekt für seine Nichte vorangetrieben hatte ${ }^{70}$.

\section{Der Hof ist ein Proteus oder: Von der Fähigkeit zur Anpassung}

Die rasche Integration in die Hofgesellschaft und die Familie der Guise, ihr gutes Verhăltnis zum Ehemann und den Damen des franzősischen Adels waren der Prinzessin durch Herkunft und Erziehung erheblich erleichtert worden. Der Hof sei wie ein Proteus, der seine Form ständig verăndere, zitiert Margarete von Valois in ihren Memoiren den Bruder, und die junge Herzogin von Aumale scheint die Fähigkeit besessen zu haben, sich den jeweiligen Gegebenheiten anzupassen. Aufgewachsen an zwei verschiedenen, teilweise konkurrierenden Höfen, beeinflußt von sprachlicher, kultureller und religiöser Vielfalt und geprägt von den Widersprlichen im Verhältnis der Eltern, war Anna den Anforderungen, die eine neue Umgebung und ein neues Leben an sie stellten, in vollem Maße gewachsen ${ }^{71}$.

Von Beginn ihrer Reise nach Frankreich an hatten die italienischen Begleiter mit Erstaunen und Bewunderung die Sicherheit zur Kenntnis genommen, mit der sich die Prinzessin in ihrer neuen Umgebung bewegte. Seine Tochter verstünde sich so gut mit ihren franzősischen Begleitern, schrieb ein Beobachter dem Herzog von Ferrara, daß der Schwiegervater und alle anderen sie

${ }^{68}$ CINZ1O, Dell' Hercole, S. 122.

${ }^{69}$ Germanico Savorgnano an Ercole d'Este, 23. Nov. 1548, Châtillon, in: ASM, Canc. duc., Amb. Francia 52.

${ }^{70}$ Ercole d'Este an Ippolito d'Este, 1. Jan. 1549, in: ASM, Casa 79.

${ }^{71}$ Marguerite de VALOIS, Mémoires, in: DIES., Mémoires et autres écrits, 1574-1614, hg. v. Éliane VIENNOT, Paris 1999, S. 13-214, hier S. 163. 
liebten. Noch bevor sie Paris erreicht hatte, war Anna zu einem Mitglied des französischen Hofes geworden: Ella si è cosi ben acomodata alli lor costumi, che V.E. diria che la fosse nata et alevata sempre in Franza, di modo che fin qua io la ho trovata francese svisu, verbo et operar. Anlaßlich ihres Einzuges in die Hauptstadt wurde die Wandlung der Prinzessin schon allein durch ihre Kleidung alla francese offenbar, und nach ihrer Ankunft bei Hofe mußten vor allem die Italiener feststellen, daß aus Ercoles Tochter eine glaubhafte Herzogin von Aumale geworden war $^{72}$. Die Metamorphose der italienischen Prinzessin erinnert an die Vorkommnisse anläßlich der Hochzeit Ludwigs XIV. mit Maria Theresia, Tochter Philipps IV. von Spanien, im Jahre 1660. Als die beiden Höfe zur Übergabe der Braut aufeinandertrafen, wurden die Anwesenden Zeugen der Verwandlung der spanischen Infantin María Teresa in die franzősische Königin Marie-Thérèse. Die Prinzessin erschien bei ihrem ersten Zusammentreffen mit dem französischen Hof in spanischer Kleidung und mußte, bevor sie ihrem zukünftigen Gemahl präsentiert wurde, in französische Tracht gekleidet werden, damit sie dem König gefalle und in seinen Augen aymable und begehrenswert erscheine. Am folgenden Tag zeigte sich die Braut wiederum nach der Mode ihrer Heimat gekleidet, doch bereits coiffée moitié à la Françoise, am Tag ihrer Hochzeit schließlich erschien sie in einem französischen Gewand, coiffée encore un peu à l'Espagnole. Den Worten des Berichterstatters zufolge wurde Maria Theresia mit jedem Tag schöner, bis sie schließlich auf vollkommene Weise dem Bild einer französischen Königin entsprach ${ }^{73}$.

Ähnlich hatte sich auch die Integration der Prinzessin aus Ferrara in die Gesellschaft des franzősischen Hofes vollzogen. Die Widmung der Übersetzung einer "Apologie contre les mesdisantz de la nation Françoise« an die junge Herzogin von Guise zeigt, daß sie schon bald als ein Mitglied dieser "Nation« angesehen wurde, und tatsächlich erwăhnt kein einziger der vielen, ihre Ankunft bei Hofe verfolgenden Gesandten und Agenten, eventuelle Probleme bei der Eingewöhnung ${ }^{74}$. Die zeitgenössischen Beobachter sahen das Geheimnis ihres Erfolgs und den Grund für das Interesse, welches der König und die Damen des Hofes ihr entgegenbrachten, in eben dieser Fähigkeit zur Anpassung, die den Eindruck entstehen ließ, Anna habe immer schon in Frankreich gelebt: Ella invero è molto ben vista et accarezzata dal re, dalla regina e da tutta questa corte, oltre tanti altri respetti per accommodarse

${ }^{72}$ Germanico Savorgnano an Ercole d'Este, 20. Nov. 1548, Dijon, in: OCCHIPINTI (Hg.), Carteggio, S. 206; Giulio Alvarotti an dens., 29. Nov. u. 5. Dez. 1548, Nogent-sur-Seine, Paris, in: ASM, Canc. duc., Amb. Francia 45; Georgio Conegrani an Francesco Gonzaga, 7. Dez. 1548, Poissy, in: ASMan, AG 641, fol. 276r.

${ }_{73}$ Abby ZANGER, Fashioning the Body Politic: Imagining the Queen in the Marriage of Louis XIV, in: Louise Olga FrADENBURG (Hg.), Women and Sovereignty, Edinburgh 1992, S. 101-120, Zitate S. 103f., 111.

${ }^{74}$ Michel Roté, Widmung seiner Übersetzung von: EQUICOLUS, Apologie. 
massimamente tanto bene a questi costumi et a questi andari, che par' propriamente che sia nata e nodreta in questa corte ${ }^{75}$. Bevor die Prinzessin jedoch in ihr neues Umfeld integriert worden war, hatte ein Prozeß der Akkulturation stattfinden müssen, der sich an ihrer Kleidung und ihrer Sprache außerte. Die Geschenke, welche der Braut von der Familie ihres zuklunftigen Gemahls gemacht worden waren, der Austausch ihres Gefolges, die Briefe, welche sie mit François vor ihrer ersten Zusammenkunft getauscht hatte, waren Zeichen einer schrittweisen Eingliederung in das Umfeld, in dem sie sich fortan bewegen würde. In einigen Bereichen des täglichen Lebens dauerte die Integration jedoch etwas länger, wie es hinsichtlich Annas erster Krankheit deutlich wird. Als sie Anfang April 1549 unter una febre lente litt und kurz darauf auch François etwas Fieber hatte, causatali, credo io, per haver' fatto troppo disordine con la predetta signora [Anna], gesundete die Prinzessin erst, als sie einen Arzt fand, der in Italien die ihr vertrauten Methoden erlernt hatte und sie alla italiana e molto bene behandeln konnte ${ }^{76}$.

Von Anfang an war Anna in die unmittelbare Umgebung des Königs und der Königin integriert, wobei vor allem die Tatsache, daß sie eine direkte Verwandte Heinrichs war, die entscheidende Rolle spielte. So schrieb etwa Alvarotti nach Ferrara, der Adel des Hofes whirde die Prinzessin in der Regel mit Madama di Umala ansprechen, doch den Umgang des Königs selbst mit der Herzogin von Aumale erläuterte er: il re, quando la chiama lei, la chiama sma cusinar, e quando mandata, come dire, a chiamarla o dirle qualche cosa, dira sa ma cusina Madama d'Umalar ${ }^{77}$. Nicht zuletzt an der Position ihrer Gemächer im Schloß von Saint-Germain kommt die herausragende Stellung der Prinzessin und der Familie ihres Gemahls zum Ausdruck. Gemeinsam mit François bewohnte sie ein Appartement im ersten Stock des Gebäudes, bestehend aus zwei Zimmern, einem kleinen Saal und zwei Garderoben, direkt zwischen den Wohnungen der Herzogin von Montmorency und der Maréchale de Saint-Andre ${ }^{78}$.

Gleich zu Beginn ihres Lebens bei Hofe hatte sich Anna beim Vater fur das Ausbleiben ihrer Briefe mit dem Hinweis entschuldigt, sie habe sowohl der Gemahlin als auch der Schwester des Königs ständig Gesellschaft leisten

${ }^{75}$ Georgio Conegrani an Sabino Callandra, 12. Dez. 1548, Poissy, in: ASMan, AG 641, fol. 286vf.

${ }^{76}$ Giulio Alvarotti an Ercole d'Este, 13. u. 28. Apr. 1549, in: ASM, Canc. duc., Amb. Francia 45; Anna d'Este an dens., 16. Apr. u. 28. Mai 1549, in: Ibid. Cart. prin. est. 1568 A/14.

${ }^{77}$ Giulio Alvarotti an Ercole d'Este, 23. Jan. 1549, Poissy, in: ASM, Canc. duc., Amb. Francia 45.

${ }^{78}$ Françoise BoudON, Monique CHATENET, Les logis du roi de France au XVI siècle, in: Jean GulllaUME (Hg.), Architecture et vie sociale. L'organisation intérieure des grandes demeures à la fin du Moyen Âge et à la Renaissance, Paris 1994, S. 65-82, hier S. 79; Monique CHATENET, Les logis des femmes à la cour des derniers Valois, in: Jan HIRSCHBIEGEL, Werner PARAVICINI (Hg.), Das Frauenzimmer. Die Frau bei Hofe in Spätmittelalter und fruher Neuzeit, Stuttgart 2000, S. 175-192, hier S. 183. 
müssen, und dem Herzog von Mantua schrieb sie, daß sie gezwungen sei, sich immer im Unfeld des Königs und der Königin aufzuhalten. Auch in den folgenden Monaten finden sich wiederholt Hinweise auf ein enges Verhältnis der Prinzessin zum Herrscherpaar. Anna sei amata et honorata da tutta la corte, $e$ dal re accarezzata e corteggiata alla stanza sua, stellte einer der Mănner des Herzogs von Ferrara fest, und drei Wochen später hieß es, von Heinrich würde sie verwöhnt und mit dessen Gattin und Schwester verbrăchte sie den größten Teil ihrer Zeit ${ }^{79}$. Besonders zu Katharina von Medici sollte sich in den folgenden Jahren ein Verhältnis entwickeln, das von Freundschaftlichkeit und großer Zuneigung geprägt war. La peur que je ay que ayés mal ist nur einer der vielen Hinweise in den Briefen der Konnigin auf ihre Sorge um die Gesundheit der Prinzessin. Sie wolle sie so oft wie möglich um sich haben, schrieb Katharina und versicherte, es gäbe niemanden, qui vous ayme myeulx. An ihren Freuden und Sorgen ließ sie die Freundin teilhaben, und die große Vertrautheit zwischen den beiden Damen ist schon allein daran zu erkennen, daß sich eine der wenigen Erwăhnungen der Geliebten ihres Gemahls durch die Königin in einem Brief an die Herzogin von Guise findet. So schrieb sie ihr, nach einem etwas melancholischen Hinweis auf das gute Verhältnis zwischen Anna und ihrem Gatten: Plet à Dyeu que je feusse aussi byen aveques le myen! Über zwei Jahrzehnte später sollte der Gesandte Englands am französischen Hof die Ebenbürtigkeit der beiden Damen auf ihre italienische Herkunft zuruckfuhren: Wenn die Königinmutter jemals ein würdiges Gegenuber gefunden habe, dann in der Herzogin von Nemours, schrieb er, und sicher lag einer der Gründe für ihre Freundschaft tatsächlich in der ăhnlichen kulturellen Prägung ${ }^{80}$.

Die Vorstellung jedoch, Anna habe Kunstler aus ihrer Heimat in großem Stil gefördert und bei Hofe ein Foyer italienscher Kultur geschaffen, kann anhand der Quellen nicht nachvollzogen werden. Der Eintritt Primaticcios in den Dienst der Guise wird wohl eher auf deren Vorliebe für italienische Künstler oder auf die vorherrschende Mode als auf den Einfluß der jungen Herzogin zurückzufuhren sein, und für die Behauptung, Niccolò dell' Abbate habe in Annas Gemächern im Hôtel de Guise Wandmalereien ausgefuhrt, finden sich keinerlei Beweise ${ }^{81}$. Anders als die dem dänischen Königshaus entstammende

79 Anna d'Este an Ercole d'Este, 29. Dez. 1548, in: ASM, Canc. duc., Cart. prin. est. $1568 \mathrm{~A} / 14$; Dies. an Francesco Gonzaga, o.D. (Anfang 1549), in: ASMan, AG 642, fol. 528r; Ferrante Trotti an Ercole d'Este, 17. Febr u. 10. März 1549, in: ASM, Canc. duc., Amb. Francia 52.

${ }^{80}$ Katharina von Medici an Anna d'Este, 18. Apr. 1551, Ende Aug. 1553, Sept. 1553, in: Catherine de MÉDICIS, Lettres, hg. v. Hector de LA FERRIĖRE, Bd. 1, Paris 1880, S. 39, 80, 82f. Der Hinweis auf Lady Fleming und Diane de Poitiers im Brief vom 18. Apr. 1551, in: Ibid. S. 39f. Valentine Dale an William Cecil, 5. März 1574, Paris, in: Calendar of State Papers. Foreign Series, of the Reign of Elizabeth, 1575-77, London 1880, ND 1966, S. $20 \mathrm{f}$.

${ }^{81} \mathrm{Zu}$ Niccolòs angeblichem Dienst für Anna d'Este: LANGLOIS, Les hôtels, S. 28. Die Behauptung, Anna habe italienische Künstler gefordert, erstmals in: ROMIER, Origines, Bd. 1, S. 71f., und in der Folge hăufig wiederholt, z.B. in: Jean-François SOLNON, La Cour de 
Anna, Kurfürstin von Sachsen, die ihre Vorliebe für dänische Stickereien, Wäsche und Hauben auch nach ihrer Heirat pflegte, scheint sich die ferraresische Prinzessin von Anfang an der Mode und Lebensweise ihrer neuen Heimat angepaßt $\mathrm{zu}$ haben ${ }^{82}$. Ein Dokument erlaubt es aber, die Verbindung zwischen Anna und in Frankreich tätigen Künstlem aus dem Land jenseits der Alpen herzustellen. Es handelt sich um einen Brief des Battista Amorevoli, der unter dem Pseudonym La Franceschina mit der Theatergruppe der Gelosi an den Hof Heinrichs III. gekommen war. Wahrscheinlich vor seiner Reise nach Venedig Anfang des Jahres 1581 schrieb er der Herzogin von Nemours einen Brief, in dem er ihre baldige Ruckkehr nach Paris mit dem Sonnenaufgang und dem Frühlingsbeginn verglich und sich in Wortspielen um ihren Namen erging. Das Schreiben endet mit einem Sonett, in dem der Schauspieler die Prinzessin als Illustrissima Aurora, alma e divina, / Lume d'Italia et gran splendor de Franza, / Vero rifugio d'ogni mia speranza bezeichnet. Die Tatsache, daß Amorevoli sich Urlaub von Anna erbat, um bei der Hochzeit des Herzogs von Mantua aufzutreten, weist darauf hin, daß die beiden tatsăchlich in einem Patronageverhältnis standen ${ }^{83}$. Schließlich ist aus der Widmung der zweiten Auflage der "Premières auvres poétiques« des Flaminio de Birague die Bedeutung zu ersehen, welche die Herzogin fur Mitglieder jener Familien hatte, die im Laufe des 16. Jahrhunderts aus Italien nach Frankreich gekommen waren. In seinem Widmungsschreiben bedankt sich der Autor bei der Prinzessin für die vertueuse magnanimité, mit der sie nicht nur ihn, sondern seine ganze Familie beehre ${ }^{84}$.

$\mathrm{Zu}$ den höfischen Verpflichtungen der Prinzessin sind auch ihre ausgedehnten Reisen zu rechnen. Der Königin zu folgen, bei Hochzeiten, Taufen, Turnieren und Festen anwesend zu sein, bei offiziellen Zeremonien den eigenen Rang und den der Familie zu repräsentieren und sich dabei immer über alles informiert zu zeigen bedeutete, stets unterwegs zu sein, sich auf unbequemen Straßen von einem Schloß zum anderen zu bewegen und an den täglichen Unterhaltungen des Hoflebens teilzunehmen. Bisognando ogni giorno andare alla caccia, hatte Anna im März 1549 erschöpft nach Ferrara geschrieben, und aus einem rund zehn Jahre später verfaßten Brief ist zu ersehen, welches

France, Paris 1987, S. 76f. Zur Förderung italienischer Künstler durch die Guise: Ian WARDROPPER, Le mécénat des Guise. Art, religion et politique au milieu du XVI siècle, in: Revue de l'art 94 (1991) S. $27-44$.

${ }^{82}$ Katrin KeLLER, Zwischen Wissenschaft und Kommerz. Das Spektrum kultureller Mittler im 16. Jahrhundert, in: Wolfgang ScHMALE (Hg.), Kulturtransfer. Kulturelle Praxis im 16. Jahrhundert, Innsbruck u.a. 2003, S. 271-286, hier S. 276.

${ }^{83}$ PICOT, La Franceschina, S. 32.

\$4 Flaminio de Birague, Widmung an Anna d'Este der »Premières auvres poétiques«, 2. Aufl., Paris 1583, fol. 6-8, hier fol. 6v, zit. n. Roland Guillot, Michel Clément, Einleitung zu: BIRAGUE, Les premières cuvres, Bd. 1, S. XLVIII. Vgl. Émile PICOT, Les Français italianisants au XVI ${ }^{e}$ siècle, Bd. 2, Paris 1907, S. $224 f$. 
Ausmaß die Anstrengungen annehmen konnten, die der Prinzessin durch ihre höfischen Verpflichtungen auferlegt waren. Das Schreiben berichtet von der Reise der französischen Königstochter Elisabeth nach Spanien:

Nous sommes venues dès avant-hier en se lieu de Bloys, au plus grand yournée qu'il a esté possible de fayre, pour la haste que le roy d'Espagne fait d'avoyr la royne sa fem[me] alla fin de se moys alla frontierre. Et an[core] que se soyt chose impossible en si peu de ta[mps], si esse que l'on fait la plus de dilligense que [l'on] peut, affin que se soit incontina $[n t]^{85}$.

Doch auch außerhalb des Dienstes für die Königin war Anna ständig unterwegs und reiste zwischen Paris und Joinville, Guise und Nanteuil hin und her. Nach der Rückkehr ihrer Mutter nach Frankreich gehörte auch Montargis zu ihrem Itinerar, und Anfang des Jahres 1561 begleitete sie den Gemahl ins Elsaß. Einen Eindruck von ihrem unsteten Leben vermittelt ein an François gerichteter Brief, der den Herzog über die Reisestationen seiner hochschwangeren Frau unterrichtete:

Estant Madame d'Aumalle jà partie de ceste ville [Paris] lors que je y arrivay, je feuz luy présenter voz lettres à Villeneufve-Sainct-George où elle fut coucher au partir de ceste dite ville. [...] Je la feuz conduire jusques auprès de Melun, et le lendemain, que estoit le jour de Sainct Nicolas, madite dame fut coucher à Fontainebleau. [...] Et a tousjours faict autant bonne chère qu'il est possible pour la grossesse où elle est ${ }^{86}$.

Auch später noch, nach ihrer zweiten Heirat, war Anna viel unterwegs, wie die große Reise zeigt, im Rahmen derer sie von September 1568 bis Januar 1569 von Paris nach Lyon und dann zurück bis nach Joinville fuhr und somit innerhalb weniger Monate eine Strecke von über tausenddreihundert Kilometern zurücklegte ${ }^{87}$.

\section{Repräsentative Aufgaben im Namen der Guise}

In den ihrer Ankunft in Frankreich folgenden Monaten und Jahren nahm Anna als Gemahlin des Herzogs von Aumale, dann von Guise, bei offiziellen Zeremonien und anderen außergewöhnlichen Ereignissen des Hoflebens stets eine privilegierte Stellung ein. Kaum eine Hochzeit oder Taufe, ein Ball oder Empfang, in dessen Beschreibung ihr Name nicht in einem Atemzug mit denen der großen Fürstinnen des Königreiches genannt worden wäre. Bei einer dieser Gelegenheiten hatte Anna die Familie der Guise zu vertreten; es ist dies das erste Mal nach ihrer Hochzeit, daß die Prinzessin bei einem offiziellen Anlaß

${ }^{85}$ Anna d'Este an Ercole d'Este, 2. März 1549, in: ASM, Canc. duc., Cart. prin. est. 1568 A/14; Dies. an Alfonso d'Este, 2. Nov. 1559, Blois, in: Ibid.

${ }^{86}$ Longnet (?) an François de Lorraine, 7. Dez. 1549, Paris, in: BnF, Fr. 20534, fol. 115 r.

${ }^{87}$ Rechnungsbuch 1569. 
einen besonders ehrenvollen Rang bekleidete. Im Februar 1549 war die Königin von ihrem vierten Kind, einem Sohn, entbunden worden, drei Monate später sollte die Taufe stattfinden. Da keiner der Taufpaten persönlich erscheinen konnte, waren Stellvertreter gewählt worden. Marie de Lorraine, Witwe des Königs von Schottland, Mutter der minderjährigen schottischen Königin Maria Stuart und ältere Schwester des Herzogs von Aumale, hatte ihre Schwägerin Anna gebeten, das Kind an ihrer Statt über das Taufbecken zu halten. Offiziell vertrat die Prinzessin zwar die Regentin von Schottland, tatsächlich war sie eines der zahlreich bei dieser Zeremonie anwesenden Mitglieder des Guise-Clans.

Die Taufe wurde im Mai in Saint-Germain-en-Laye gefeiert. Annas Platz bei dem der Zeremonie vorausgehenden Festbankett befand sich zur Rechten und in fast unmittelbarer Nähe Heinrichs II., zwischen ihr und dem Herrscher saßen lediglich dessen geliebte Schwester Margarete und die siebenjährige Königin von Schottland. Die Herzogin von Aumale war somit noch vor dem portugiesischen Gesandten, der seinen König als Taufpaten zu vertreten hatte, plaziert. Nach dem Festessen und dem anschließenden Ball brachte man den kleinen Prinzen, der den Namen Louis erhalten sollte, im Triumphzug zur Schloßkirche. Gehalten wurde der Säugling von dem Gesandten aus Portugal, zu dessen Rechter Claude de Lorraine als Stellvertreter des zweiten Paten und zu dessen Linker Anna d'Este einherschritten. Sechs der anwesenden Herren waren direkte Angehörige der Familie der Guise, Onkel oder Brüder des Herzogs von Aumale. Sie trugen die für die Zeremonie notwendigen Gegenstände in die Kirche; selbst der kaum zwölfjährige René durfte einen Zipfel des goldenen Tuches, auf das der königliche Prinz gebettet war, halten. Auch einige der Damen waren mit den Guise verwandt oder verschwägert, so etwa Diane de Poitiers, die eine ihrer Töchter mit einem Bruder von François de Lorraine verheiratet hatte, oder dessen direkte Nichte, die kleine schottische Königin Maria Stuart ${ }^{88}$.

Die Bedeutung der Guise, ihre Stellung bei Hofe und die Verflechtung der Familie in das unmittelbare Umfeld des Königs waren für alle Gäste der Tauffeier offensichtlich. Besonders Constantino de Bragança, der aus Portugal angereiste Gesandte, zeigte sich von dieser Machtdemonstration beeindruckt. Während der Herzog von Ferrara der Königin kostbare Gaben hatte überreichen lassen, beschenkte der Portugiese nur zwei Damen des Hofes, wie Anna dem Vater berichtete: Lo imbasciatore di Portogallo non ha ancora fatto niuno presente se non a madama di Valentinois et a me, che si ha donatto a

\footnotetext{
${ }^{88}$ L'ordre et triumphe du baptesme de très hault et puissant prince Monseigneur Louis second, filz de France, duc d'Orléans, o.D. (19. Mai 1549), Kopie des 17. Jh., in: BnF, Fr. 4324, fol. 14-20. Vgl. Théodore GoDEFroY, Denys GODEFroY (Hg.), Le Cérémonial Francois, Bd. 2, Paris 1649, S. 149-156; Jacques-Auguste de THOU, Histoire universelle, Bd. 1, Basel 1742, S. 494.
} 
ciascheduno dua para di guanti, del che se ne sono tutti beffati. Die Befürchtung des Gesandten, angesichts der Kostbarkeiten, die der Königin von einem italienischen Herzog überbracht worden waren, bei der Überreichung des Geschenks seines Königs eine Blamage zu erleiden, fuhrte zu einer merkwürdigen Situation. Die Herzogin von Valentinois als einflußreichste Dame Frankreichs und Anna als Repräsentantin einer der mächtigsten Familien waren von Don Constantino bedacht worden, während Katharina von Medici, Königin und Mutter des jungen Prinzen, ıvergessen` worden war - kein Wunder, daß sich die Damen und Herren des Hofes über den jungen Mann lustig machten ${ }^{89}$.

Es sollte dies nicht das letzte Mal gewesen sein, daß Anna bei der Taufe eines königlichen Prinzen als Patin fungierte. Im folgenden Jahr hielt sie im Namen ihrer Mutter den späteren Karl IX. über das Taufbecken, 1554 bekam das achte Kind des Herrscherpaares, der spätere François, Herzog von Alençon, dann von Anjou, von Anna den Namen ihres Vaters, Hercule, und wahrend der Taufe der Prinzessin Victoire, welche die ersten Lebenswochen nicht uberleben sollte, war ihre Anwesenheit vorgesehen pour découvrir le lict $^{90}$. Nicht weniger ehrenvoll war die Aufgabe, die der Herzogin von Aumale im Juni 1549, anläßlich der Krönung der Königin in Saint-Denis, zufiel. Nach der Krönungszeremonie wurde die Messe gefeiert. Am Altar erhielt Katharina ein goldenes Brot, ein silbernes Brot, einen Becher Wein und eine Kerze gereicht. Die Namen der Damen, die ihrer Königin bei diesem Vorgang assistierten, verraten den Rang, den Anna bei Hofe bekleidete. Antoinette de Bourbon, Herzogin von Guise, überreichte Katharina den goldenen Brotlaib, Marguerite de Bourbon, Herzogin von Nevers, den silbernen; die Kerze, bestückt mit dreizehn goldenen Münzen, wurde von Diane de Poitiers, Herzogin von Valentinois, überreicht und der Wein von Anna, der jungen Herzogin von Aumale. Bei dem festlichen Einzug der Königin in Paris eine Woche später ritt die Prinzessin auf einem weißen Zelter mit silberner Schabracke, wie die anderen Damen war sie ganz in weiß gekleidet und mit Edelsteinen geschmückt. Auf dem Kopf trug sie eine Krone, und ihre Schleppe war so lang, daß sie von einem neben ihrem $P$ ferd gehenden Herrn getragen werden mußte ${ }^{91}$.

${ }^{89}$ Anna d'Este an Ercole d'Este, 28. Mai 1549, in: OCCHIPINTI (Hg.), Carteggio, S. 219; Niccolò Bendidio an dens., 7. Juni 1549, in: Ibid. S. $220 \mathrm{f}$.

90 Zu Karl IX.: Renée de France an Heinrich II., 26. Juli (1550), Ferrara, in: BnF, Fr. 20453, S. 121; Giulio Alvarotti an Ercole d'Este, 2. Sept. 1550, Poissy, in: OCCHIPINTI (Hg.), Carteggio, S. 251f. Zu François de Valois: Naissances des enfans du roy Henry de bonne mémoyre, in: Louis PARIS (Hg.), Négociations, lettres et pièces diverses relatives au règne de François II, tirée du portefeuille de Sébastien de l'Aubespine, évêque de Limoges, Paris 1841, S. 894. Zur Taufe von Victoire de France im Jahre 1556: GoDEFROY (Hg.), Cérémonial François, S. 157.

${ }^{91}$ L'Ordre et forme qui a esté tenue au Sacre et Couronement de treshaulte et tresillustre Dame, Madame Catharine de Medicis, Royne de France, faict en l'Eglise Monseigneur 
Hatten sich die Träume des Herzogs von Ferrara, seine älteste Tochter zur Königin zu machen, auch nicht wirklich erfült, so mußte Ercole bei der Lektüre der Berichte seiner Gesandten und Agenten doch eine gewisse Genugtuung verspürt haben. In einem anläßlich der Abreise der Prinzessin aus ihrer Heimatstadt verfaßten Lied Cyprians de Rore heißt es, Anna würde schon bald nach Ferrara zuruckkehren, wenn nicht in persona, so doch mittels ihres Rufes und Ruhmes: Tost sera si grande sa puissance, / Que la verrés yci et en tous lieux. Und Anna kehrte als Königin nach Ferrara zurück, zwar nicht tatsächlich, aber doch durch die Briefe der Berichterstatter des Herzogs und der Herzogin. Wie eine Königin war sie in Paris empfangen worden, und ihre Hochzeitsfeier war so prăchtig ausgefallen wie die einer Königin. Stets war die Prinzessin königlich gekleidet und geschmückt, und der Rang, den sie bei Hofe einnahm, ließ nichts zu wünschen übrig. Darüber hinaus hatte Heinrich dem Herzog von Aumale zugesichert, che stia di buon animo che lo fara grande - Was mehr hătte Ercole sich für seine Tochter wünschen können, was mehr Renée de France? War die Trennung von ihrer italienischen Heimat für die Prinzessin auch schmerzhaft gewesen, so war ihre Integration in die französische Adelsgesellschaft geglückt. Im Laufe ihrer Reise über die Alpen und durch Frankreich hatte Anna reale und symbolische Grenzen überschritten, und während der ersten Wochen und Monate ihres Lebens bei Hofe war sie von einer Objektrolle, die sie wăhrend der Verhandlungen und Gespräche im Vorfeld der Heirat und noch bei ihrem ersten Zusammentreffen mit dem zukünftigen Gemahl innegehabt hatte, in eine Subjektrolle gewechselt. Die Integration der jungen Frau in die Hofgesellschaft war so reibungslos verlaufen, wie es bei kaum einer anderen ihrer Standesgenossinnen der Fall war. Zu verdanken war dies ihrer Herkunft und ihrer Erziehung, Voraussetzungen, die Anna schon zur Herzogin von Aumale hatten werden lassen, bevor sie an ihrem Hochzeitstag die Schloßkirche von Saint-Germain betrat ${ }^{92}$.

Sainct-Denys en France, o.O. o.J. [1549], in: BnF, Fr. 3107, S. 107-125; L'Ordre de l'entrée de la Reine à Paris, o.O. o.J. [1549], in: BnF, Fr. 3107, S. 85-105; Giulio Alvarotti an Ercole d'Este, 18. Juni 1549, Paris, in: OCCHIPINTI (Hg.), Carteggio, S. 226-228.

${ }^{92}$ Cyprian de Rore, En voz adieux - Hellas, comment, in: RORE, Opera, Bd. 8, S. 39-43; Giulio Alvarotti an Ercole d'Este, 23. Jan. 1549, Poissy, in: ASM, Canc. duc., Amb. Francia 45. 
